TRANSACTIONS OF THE

AMERICAN MATHEMATICAL SOCIETY

Volume 353, Number 9, Pages 3661-3683

S 0002-9947(01)02662-9

Article electronically published on April 24, 2001

\title{
SIEGEL DISCS, HERMAN RINGS AND THE ARNOLD FAMILY
}

\author{
LUKAS GEYER
}

\begin{abstract}
We show that the rotation number of an analytically linearizable element of the Arnold family $f_{a, b}(x)=x+a+b \sin (2 \pi x)(\bmod 1), a, b \in \mathbb{R}$, $0<b<1 /(2 \pi)$, satisfies the Brjuno condition. Conversely, for every Brjuno rotation number there exists an analytically linearizable element of the Arnold family. Along the way we prove the necessity of the Brjuno condition for linearizability of $P_{\lambda, d}(z)=\lambda z(1+z / d)^{d}$ and $E_{\lambda}(z)=\lambda z e^{z}, \lambda=e^{2 \pi i \alpha}$, at 0 . We also investigate the complex Arnold family and classify its possible Fatou components. Finally, we show that the Siegel discs of $P_{\lambda, d}$ and $E_{\lambda}$ are quasidiscs with a critical point on the boundary if the rotation number is of constant type.
\end{abstract}

\section{INTRODUCTION}

The first part of this paper studies the problem of linearizability in some special families of functions. We investigate the analytic functions $P_{\lambda, d}(z)=\lambda z(1+z / d)^{d}$ and $E_{\lambda}(z)=\lambda z e^{z}$ for $\lambda=e^{2 \pi i \alpha}, \alpha \in \mathbb{R} \backslash \mathbb{Q}$. They have an irrationally indifferent fixed point at 0 and in the case of linearizability 0 is the center of a Siegel disc. By a result of Brjuno and Rüssmann these functions are linearizable for $\alpha \in \mathcal{B}$ where $\mathcal{B}$ is the set of Brjuno numbers. It is defined by $\mathcal{B}:=\left\{\alpha \in \mathbb{R} \backslash \mathbb{Q} \mid \sum q_{n}^{-1} \log q_{n+1}<\infty\right\}$ where $\left(p_{n} / q_{n}\right)$ are the convergents of $\alpha$, coming from the continued fraction development. Yoccoz showed that the Brjuno condition is also necessary for linearizability of quadratic polynomials Y Yo1]. We show the necessity of the Brjuno condition for the families $P_{\lambda, d}$ and $E_{\lambda}$. It is still an open problem whether a polynomial of degree $\geq 3$ could have a Siegel disc with non-Brjuno rotation number [Do]. It is likely that our techniques can be extended from the special families $P_{\lambda, d}$ and $E_{\lambda}$ to more general families of maps. However, it is quite clear that limitations of our methods prevent them from solving the general question.

In the second part of the paper these results are transferred to circle diffeomorphisms by means of quasiconformal surgery techniques. It turns out that $E_{\lambda}$ corresponds to the well-known Arnold family $f_{a, b}(x)=x+a+b \sin (2 \pi x)(\bmod 1)$, $a, b \in \mathbb{R}, 0<b<1 /(2 \pi)$. The result we get is the following: If $f_{a, b}$ is analytically linearizable, then the rotation number $\rho\left(f_{a, b}\right)$ is a Brjuno number. Conversely, for every Brjuno rotation number there exists an analytically linearizable element of

Received by the editors December 18, 1998 and, in revised form, December 12, 1999.

2000 Mathematics Subject Classification. Primary 30D05; Secondary 58F03, 58F08.

Key words and phrases. Arnold family, standard family, linearization, Herman rings, circle diffeomorphisms.

The author wishes to thank "Studienstiftung des deutschen Volkes" and DAAD for financial support. 
the Arnold family. The family $P_{\lambda, d}$ corresponds to a family of Blaschke products (with poles in the unit disc) for which the same result holds.

We proceed to investigate the complex Arnold family $F_{a, b}(z)=e^{2 \pi i a} z e^{\pi b(z-1 / z)}$ which was investigated by Fagella ([Fa1], [Fa2]) before (for other values of the parameters). We show that the Fatou set of $F_{a, b}$ consists of at most one periodic cycle and its preimages. The periodic cycle is either an Arnold-Herman ring containing the unit circle $\mathbb{T}$ or a cycle of attracting or parabolic domains associated to a periodic orbit on the unit circle.

The last section deals with the boundaries of the Siegel discs of $P_{\lambda, d}$ and $E_{\lambda}$, and the boundaries of the Arnold-Herman rings of $F_{a, b}$. Under the assumption that the rotation number is of constant type, i.e. has bounded coefficients in the continued fraction expansion, we show that the boundaries of the Siegel discs are quasicircles containing a critical point of the map.

We require some familiarity with the theory of complex dynamics and quasiconformal mappings. The first topic is covered in the monographs $\mathrm{Be}, \mathrm{CG}$, $\mathrm{Mi}$, St. For quasiconformal maps we refer the reader to [Ah1], BI] and [LV]. For circle diffeomorphisms the basic reference is the excellent monograph [He1, one may also consult $\mathrm{MS}$. Some background on the connections between irrationally indifferent fixed points and circle diffeomorphisms and an exposition of the new techniques of Yoccoz and Pérez-Marco can be found in [PM1] which also contains a list of references.

\section{Preliminaries}

Let $U \subseteq \mathbb{C}$ be open and $f: U \rightarrow \mathbb{C}$ of Sobolev class $W_{\text {loc }}^{1,2}$, i.e. with distributional first derivatives which are locally square-integrable. We say that $f$ is $K$-quasiregular $(K \geq 1)$ if $|\bar{\partial} f| \leq(K-1) /(K+1)|\partial f|$ almost everywhere. (Here $\partial$ and $\bar{\partial}$ are short notations for $\partial / \partial z$ and $\partial / \partial \bar{z}$, respectively.) If $f$ is $K$-quasiregular for some $K \geq 1$, we say that $f$ is quasiregular. Quasiregular maps are continuous and almost everywhere differentiable. A map is analytic iff it is 1-quasiregular. If $f$ is $(K$-)quasiregular and injective, then $f$ is called $(K-)$ quasiconformal. Non-constant quasiregular mappings are open and satisfy $\partial f \neq 0$ a.e. Thus we may define the complex dilatation $\mu_{f}:=\bar{\partial} f / \partial f$ of $f$. This function $\mu_{f}$ is measurable and $\left\|\mu_{f}\right\|<1$ where $\|\cdot\|$ denotes the essential supremum. We call each measurable function $\mu: U \rightarrow \mathbb{C}$ with $\|\mu\|<1$ a Beltrami coefficient in $U$. Fundamental is the Measurable Riemann Mapping Theorem due to Morrey, Bojarski, Ahlfors and Bers. It says that for any Beltrami coefficient $\mu$ there is a quasiconformal map with complex dilatation $\mu$. This implies that a quasiregular map $f$ can always be written as $g \circ h$ where $g$ is analytic, $h$ is quasiconformal and $\mu_{f}=\mu_{h}$ a.e. In the following we will always assume quasiregular maps to be non-constant.

If $f: U \rightarrow V$ is quasiregular and $\mu: V \rightarrow \mathbb{C}$ is a Beltrami coefficient, we define the pull-back of $\mu$ under $f$ by

$$
f^{*} \mu:=\frac{\bar{\partial} f+(\mu \circ f) \overline{\partial f}}{\partial f+(\mu \circ f) \partial \bar{f}} .
$$

This is the dilatation of $g \circ f$ where $g$ is any quasiregular map with $\mu_{g}=\mu$. The pull-back is again a Beltrami coefficient.

The important fact about the pull-back is the following; see also Figure 1. 


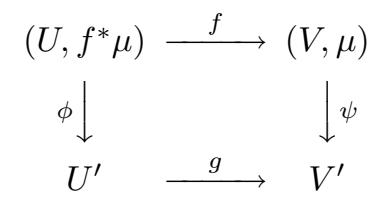

Figure 1. Situation of Proposition [2.1. The map $g$ is analytic and it is convenient to think of $\mu$ as a complex structure on $V$, different from the standard structure. In this interpretation, $f^{*} \mu$ is the unique structure which makes $f:\left(U, f^{*} \mu\right) \rightarrow(V, \mu)$ analytic and $\phi$ and $\psi$ are charts.

Proposition 2.1. Let $f: U \rightarrow V$ be quasiregular and $\mu: V \rightarrow C$ a Beltrami coefficient. Let $\phi: U \rightarrow \mathbb{C}$ and $\psi: V \rightarrow \mathbb{C}$ be quasiconformal maps with $\mu_{\psi}=\mu$ and $\mu_{\phi}=f^{*} \mu$. Then the mapping $g:=\psi \circ f \circ \phi^{-1}: U^{\prime} \rightarrow V^{\prime}$ is analytic, where $U^{\prime}=\phi(U)$ and $V^{\prime}=\psi(V)$.

Proof. As a quasiregular map with dilatation $f^{*} \mu$, the map $\psi \circ f$ can be written as $h \circ \tilde{\phi}$ with $\mu_{\tilde{\phi}}=f^{*} \mu$. Since $\mu_{\phi}=f^{*} \mu$, we get that $\tilde{\phi} \circ \phi^{-1}$ is conformal, hence $g=\psi \circ f \circ \phi^{-1}=h \circ \tilde{\phi} \circ \phi^{-1}$ is analytic.

If $f$ is analytic, the formula for the pull-back reduces to $f^{*} \mu=(\mu \circ f) \cdot(\overline{\partial f} / \partial f)$. This implies $\left|f^{*} \mu\right|=|\mu \circ f|$, i.e. pulling back by analytic functions does not increase the dilatation. Furthermore, the pull-back is analytic, i.e. if $\mu_{a}$ depends analytically on $a$, then $f^{*} \mu_{a}$ depends analytically on $a$, too.

We end this brief account with a powerful tool which we will need several times. The proof of the following "generalized qc-lemma" can be found in Ge1], [Hi] and $[\mathrm{Su}$. The original qc-lemma goes back to Shishikura [Sh].

Generalized qc-Lemma. Let $G \subset \hat{\mathbb{C}}$ be a domain and $f: G \rightarrow G$ a quasiregular map. Assume that all iterates $f^{n}$ are $K$-quasiregular with fixed $K$. Then there exists a quasiconformal map $\phi: G \rightarrow H$ such that $\phi f \phi^{-1}: H \rightarrow H$ is analytic.

\section{The families $P_{\lambda, d}$ AND $E_{\lambda}$}

In this section we establish the necessity of the Brjuno condition for linearizability in the families $P_{\lambda, d}(z)=\lambda z(1+z / d)^{d}$ and $E_{\lambda}(z)=\lambda z e^{z}$ which is the locally uniform limit of $P_{\lambda, d}$ for $d \rightarrow \infty$. In our proof we make essential use of a technique developed in [PM2]. If there is a Siegel disc, we show stability of the dynamics (in particular, of the Siegel disc) under certain perturbations. Using a kind of maximum principle, we conclude that the quadratic polynomial has a Siegel disc, too. Our perturbations will be constructed by some quasiconformal surgery procedure.

3.1. Uniform linearizability. The following proposition is essentially due to Pérez-Marco (contained in [PM2, IV.2] in a slightly weaker form; see also [Ge2]). We say that a family $f_{a}(z)=\lambda z+\sum_{k=2}^{\infty} c_{k}(a) z^{k}, \lambda=e^{2 \pi i \alpha}$, is uniformly linearizable for $a \in U$ if the $f_{a}$ all have Siegel discs of uniform size, i.e. there exists $r>0$ and a family of conformal maps $\phi_{a}: D_{r}(0) \rightarrow \mathbb{C}$ with $\phi_{a}(0)=0, \phi_{a}^{\prime}(0)=1$ and $f_{a}\left(\phi_{a}(z)\right)=\phi_{a}(\lambda z)$. 
Proposition 3.1. If $f_{a}(z)=f(z)+c(a) z^{2} g(z)$ if uniformly linearizable for $|a| \leq \varepsilon$, where $f, g$ and $c$ are analytic, $f(0)=c(0)=0, f^{\prime}(0)=\lambda=e^{2 \pi i \alpha}, \alpha \in \mathbb{R} \backslash \mathbb{Q}$, $g(0) \neq 0$ and $c$ is non-constant, then the quadratic polynomial $P_{\lambda}(z)=\lambda z+z^{2}$ is linearizable and $\alpha \in \mathcal{B}$.

Remark. If one was to transfer our proof to more general families of maps, an important step would be to generalize this proposition. The main idea behind its applications is that one can embed a linearizable map in some (non-trivial) oneparameter family of linearizable maps. In our examples we can explicitly calculate these perturbations which are of the desired (simple) form. For general families of maps one would maybe have to weaken the assumptions to allow for more complicated perturbations. The main idea behind the proof of this proposition (and related ones) is to go from "small" perturbations to "large" ones and finally apply a kind of maximum principle to get linearizability for the "infinitely large" perturbed map which is the quadratic polynomial in our case. This method of proof seems hard to implement if very general analytic perturbations are allowed. However, it might be worthwhile to see how far one can push this technique. On the other hand, it is easy to see that there are "rigid" maps which cannot easily be embedded in any non-trivial family in a controlled way. One example is $P(z)=z^{3}+c$ where $c$ is chosen such that $P$ has an irrationally indifferent fixed point. In general, if all critical points belong to the Julia set and there is no invariant line field on the Julia set, the space of quasiconformal deformations reduces to a point and our methods cannot be applied.

Proof. We may assume that $c(a)=a$ because non-constant analytic maps are open, so $c\left(D_{\varepsilon}(0)\right)$ contains some disc $D_{\varepsilon^{\prime}}(0)$ and the family of maps $f(z)+a z^{2} g(z)$ for $|a| \leq \varepsilon^{\prime}$ is uniformly linearizable. We may also assume $g(0)=1$. Let

$$
\begin{aligned}
h_{b}(z) & =\frac{1}{b} f_{1 / b}(b z) \\
& =\frac{f(b z)}{b}+z^{2} g(b z) .
\end{aligned}
$$

Due to the assumptions, for $b \in B:=\{1 / \varepsilon \leq|b| \leq 2 / \varepsilon\}$ the conformal radius of the Siegel disk of $f_{1 / b}$ is bounded below by some constant $r>0$, so the function $h_{b}$ has Siegel discs of conformal radius $\geq r \varepsilon / 2$ for $b \in B$. Define $\delta:=r \varepsilon / 8$. By the Koebe 1/4-Theorem the disc $D_{\delta}(0)$ is contained in the Siegel disc of $h_{b}$ for $b \in B$, implying that there are no periodic points of $h_{b}$ in $D_{\delta}(0) \backslash\{0\}$. We will show that $P_{\lambda}$ is linearizable, moreover, its Siegel disc contains $D_{\delta}(0)$. This is accomplished by a kind of maximum principle for linearization, which already appears in Yo1 in a special case. Yoccoz used the formal linearizing power series and the classical maximum principle in order to show it. We will follow PérezMarco's idea of applying Hartogs' theorem.

Assume that the claim is false. Then there are points in $J\left(P_{\lambda}\right) \cap D_{\delta}(0)$. Periodic points are dense in the Julia set and there are no isolated points in $J\left(P_{\lambda}\right)$, so there exists a point $z_{0} \in D_{\delta}(0) \backslash\{0\}$ and a number $q \geq 1$ with $P_{\lambda}^{q}\left(z_{0}\right)=z_{0}$. Define a holomorphic function of two variables by

$$
F(b, z)=\frac{h_{b}^{q}(z)-z}{z}
$$

for $|b| \leq 2 / \varepsilon,|z| \leq \delta$. This function is continuous and satisfies $\left.F(b, 0)=\lambda^{q}-1 \neq 0\right)$. By the first part of the proof, $F$ has no zeros in $(b, z) \in B \times D_{\delta}(0)$. By continuity 
of $F$ we may choose $\rho>0$ such that $F$ has no zeros for $(b, z) \in D_{2 / \varepsilon}(0) \times D_{\rho}(0)$. The function $F$ is thus analytic and non-zero in

$$
\left(B \times D_{\delta}(0)\right) \cup\left(D_{2 / \varepsilon}(0) \times D_{\rho}(0)\right) .
$$

By GF, Satz I.5.5] the function $1 / F$ has an analytic extension (without poles) from this domain to $D_{2 / \varepsilon}(0) \times D_{\delta}(0)$, which has to coincide with $1 / F$ by the permanence principle. But $h_{0}=P_{\lambda}$, so $F$ has a zero and $1 / F$ has a pole at $\left(0, z_{0}\right)$. This contradiction proves the proposition.

3.2. The polynomials $P_{\lambda, d}$. Now we have collected the prerequisites and proceed to prove our main theorems in this section, starting with the polynomial case.

Theorem 3.2. Let $\lambda=e^{2 \pi i \alpha}, \alpha \in \mathbb{R} \backslash \mathbb{Q}$, and $d \geq 2$ be an integer. Then the polynomial $P_{\lambda, d}(z)=\lambda z(1+z / d)^{d}$ is linearizable at 0 iff $\alpha \in \mathcal{B}$.

Before starting with the proof, let us first give an immediate implication.

Corollary 3.3. Let $\lambda=e^{2 \pi i \alpha}, \alpha \in \mathbb{R} \backslash \mathbb{Q}$, and $d \geq 2$ be an integer. Then the polynomial $\hat{P}_{\lambda, d}(z)=\lambda z\left(1+z^{d}\right)$ is linearizable at 0 iff $\alpha \in \mathcal{B}$.

Proof. This follows immediately from the theorem as $P_{\lambda, d}$ and $\hat{P}_{\lambda, d}$ are semiconjugate via $h_{d}(z)=d z^{d}$, i.e. $h_{d}\left(\hat{P}_{\lambda, d}(z)\right)=P_{\lambda, d}\left(h_{d}(z)\right)$. If $U$ is a neighborhood of 0 with $\hat{P}_{\lambda, d}(U) \subseteq U$ then $U^{\prime}=h_{d}(U)$ is a neighborhood of 0 with $P_{\lambda, d}\left(U^{\prime}\right) \subseteq U^{\prime}$ and vice versa. So if one of the two functions is linearizable, then the other one has a forward invariant neighborhood of 0 and thus is linearizable, too.

Proof of the Theorem. If $\alpha \in \mathcal{B}$, then $P_{\lambda, d}$ is linearizable by Brjuno's result. Let us assume that $P=P_{\lambda, d}$ is linearizable. For the rest of the proof, $\lambda$ and $d$ are kept fixed and the dependence on them will be suppressed in the notation. The general strategy of the proof is to modify $P$ in order to get an analytic family of quasiregular maps $P_{a}$ with $P_{0}=P$. Then an analytic family of $P_{a}$-invariant Beltrami coefficients $\mu_{a}$ is constructed, i.e. $\mu_{a}: \mathbb{C} \rightarrow \mathbb{C}$ with $\left\|\mu_{a}\right\|<1$ and $P_{a}^{*} \mu_{a}=\mu_{a}$. By the Measurable Riemann Mapping Theorem we get an analytic family of normalized quasiconformal maps $\phi_{a}$ with complex dilatations $\mu_{a}$. Using the $P_{a}$-invariance of $\mu_{a}$, we obtain analytic maps $Q_{a}=\phi_{a} \circ P_{a} \circ \phi_{a}^{-1}$. With some additional effort, using the special form of $P$ and our perturbations together with the analyticity in $a$, we show that the $Q_{a}$ are really the desired perturbations to apply Proposition 3.1 .

Let us go to the details: Due to the assumption, $P$ has a Siegel disc $S$ centered at 0 and $P^{-1}(S)=S \cup S^{\prime}$ where $S^{\prime}$ is the component of the Fatou set containing $-d$. (This follows from $P^{-1}(0)=\{0,-d\}$.) Choose $\varepsilon>0$ such that $D_{2 \varepsilon}(-d) \subset S^{\prime}$ and an arbitrary $C^{\infty}$-function $\eta:[0, \infty) \rightarrow[0,1]$ with $\eta(t)=1$ for $t \leq 1$ and $\eta(t)=0$ for $t \geq 4$. For $a \in \mathbb{C}$ we define

$$
P_{a}(z)=P(z)+a \eta\left(\frac{|z+d|^{2}}{\varepsilon^{2}}\right) .
$$

Then each $P_{a}$ is a $C^{\infty}$-function, $P_{a}(z)=P(z)$ for $|z+d| \geq 2 \varepsilon$ and $P_{a}(z)=P(z)+a$ for $|z+d| \leq \varepsilon$. Furthermore, $P_{a}$ depends analytically on $a$ with $P_{0}=P$. We want to show that $P_{a}$ is quasiregular for suitably small $|a|$.

Define $A:=\{z \in \mathbb{C}|\varepsilon \leq| z+d \mid \leq 2 \varepsilon\}$. Then $P_{a}$ is analytic, therefore quasiregular, in $\mathbb{C} \backslash A$. The map $P_{a}$ is smooth, hence also in $W_{\text {loc }}^{1,2}$. It remains to calculate 
the derivatives. We get

$$
\begin{aligned}
\partial P_{a}(z) & =P^{\prime}(z)+a \eta^{\prime}\left(\frac{|z+d|^{2}}{\varepsilon^{2}}\right) \frac{\overline{z+d}}{\varepsilon^{2}}, \\
\bar{\partial} P_{a}(z) & =a \eta^{\prime}\left(\frac{|z+d|^{2}}{\varepsilon^{2}}\right) \frac{z+d}{\varepsilon^{2}} .
\end{aligned}
$$

Now $\eta^{\prime}$ is continuous with compact support, thus $C:=\sup \left|\eta^{\prime}(t)\right|<\infty$. Also there are no critical points of $P$ in $A$. This is obvious by calculation and assuming $\varepsilon$ small enough but also immediate from results of complex dynamics, as $-d \notin A$ and the orbit of the other critical point needs to accumulate in the boundary of the Siegel disc, whereas all forward iterates of $A$ are bounded away from $\partial S$. We get $c:=\inf _{z \in A}\left|P^{\prime}(z)\right|>0$ by compactness of $A$, so

$$
\begin{aligned}
& \left|\bar{\partial} P_{a}(z)\right| \leq|a| C \frac{1}{\varepsilon^{2}} 2 \varepsilon \\
& \left|\partial P_{a}(z)\right| \geq c-|a| C \frac{1}{\varepsilon^{2}} 2 \varepsilon
\end{aligned}
$$

for $z \in A$. Choosing $\delta:=\varepsilon c /(6 C)$ we get

$$
\begin{aligned}
& \left|\bar{\partial} P_{a}(z)\right| \leq \frac{c}{3}, \\
& \left|\partial P_{a}(z)\right| \geq \frac{2 c}{3}
\end{aligned}
$$

for $|a| \leq \delta$ and $z \in A$. This implies $\left|\bar{\partial} P_{a}\right| \leq\left|\partial P_{a}\right| / 2$, so all $P_{a}$ for $|a| \leq \delta$ are 3 -quasiregular. This completes the first step of the proof. Observe that $P_{a}$ for $a \neq 0$ is dynamically distinguishable from $P_{0}$ : For $P_{0}$ the irrationally indifferent fixed point is a critical value, for $a \neq 0$ it is not. Apart from this, all the $P_{a}$ have a similar dynamical behavior: They all have Siegel discs centered at 0, two critical points, one of them with multiplicity $d-1$, mapped into the Siegel disc, and a simple one whose orbit closure contains the boundary of the Siegel disc.

The next step is to construct the invariant Beltrami coefficients $\mu_{a}$. This is done by a standard procedure using the fact that every orbit enters $A$ at most once and then stays in $S$ where all $P_{a}$ are analytic. We cannot use the generalized qc-lemma here because we need the analytic dependence of the invariant Beltrami coefficient on the parameter $a$. This does not follow in general, so we have to construct our Beltrami coefficient "by hand". (This is in fact the original construction of Shishikura in an easy case of his qc-lemma.)

We define $\mu_{a}$ pointwise by

$$
\begin{aligned}
\mu_{a}(z) & := \begin{cases}\left(P^{n}\right)^{*} \mu_{P_{a}}(z) & \text { for } P^{n}(z) \in A, \\
0 & \text { if no such } n \text { exists }\end{cases} \\
& =\sum_{n=0}^{\infty}\left(P^{n}\right)^{*} \mu_{P_{a}}(z) I_{P^{-n}(A)}(z) .
\end{aligned}
$$

The last expression $\left(I_{M}(z)\right.$ denotes the indicator function of the set $\left.M\right)$ shows that $\mu_{a}$ is measurable. The pull-back is analytic, hence $\mu_{a}$ is analytic in $a$. We claim that $\mu_{a}$ is $P_{a}$-invariant. We may write

$$
\mu_{a}(z)= \begin{cases}\left(P_{a}^{n}\right)^{*} \mu_{P_{a}}(z) & \text { for } P_{a}^{n}(z) \in A, \\ 0 & \text { if no such } n \text { exists }\end{cases}
$$


because $P$ and $P_{a}$ agree on all preimages of $A$. Then

$$
\begin{aligned}
P_{a}^{*} \mu_{a}(z) & = \begin{cases}P_{a}^{*}\left(P_{a}^{n}\right)^{*} \mu_{P_{a}}(z) & \text { for } P_{a}^{n}\left(P_{a}(z)\right) \in A, \\
P_{a}^{*} 0(z) & \text { if no such } n \text { exists }\end{cases} \\
& = \begin{cases}\left(P_{a}^{n+1}\right)^{*} \mu_{P_{a}}(z) & \text { for } P_{a}^{n+1}(z) \in A, \\
\mu_{P_{a}}(z) & \text { for } z \in A, \\
0 & \text { if } P_{a}^{n}(z) \notin A \text { for all } n \geq 0\end{cases} \\
& =\mu_{a}(z) .
\end{aligned}
$$

By the Measurable Riemann Mapping Theorem we get an analytic family of quasiconformal maps $\phi_{a}: \mathbb{C} \rightarrow \mathbb{C}$ with $\phi(0)=0, \phi(-d)=-d$ and $\mu_{\phi_{a}}=\mu_{a}$. All maps $\phi_{a}$ are analytic in $S$. Define $Q_{a}:=\phi_{a} \circ P_{a} \circ \phi_{a}^{-1}$. Due to the $P_{a}$-invariance of $\mu_{a}$, $Q_{a}$ is analytic. The map $Q_{a}$ is a proper map of the plane onto itself of topological degree $d+1$, hence a polynomial of degree $d+1$. The conjugating function $\phi_{a}$ is analytic near 0 , so $Q_{a}(0)=0, Q_{a}^{\prime}(0)=\lambda$. Furthermore, $Q_{a}$ has a $(d-1)$-fold critical point at $-d$ and a simple critical point at $c_{a}$. Define $v_{a}:=Q_{a}(-d)$. Then $v_{0}=0$ and $a \mapsto v_{a}=\phi_{a}(a)$ is analytic and non-constant for small $|a|$. Define $h_{a}:=Q_{a}-P$. Then $h_{a}$ is a polynomial of degree $d+1$ with $h_{a}(0)=0, h_{a}^{\prime}(0)=0$ and $h_{a}^{\prime}(-d)=0$ with multiplicity $\geq d-1$. Thus

$$
\begin{aligned}
h_{a}^{\prime}(z) & =C_{a} z\left(1+\frac{z}{d}\right)^{d-1} \\
h_{a}(z) & =C_{a} \int_{0}^{z} \zeta\left(1+\frac{\zeta}{d}\right)^{d-1} d \zeta \\
& =C_{a} z^{2} h(z)
\end{aligned}
$$

where $h$ is a polynomial of degree $d-1$ with $h(0) \neq 0$. We want to show that $C_{a}$ is analytic in $a$. We have

$$
\begin{aligned}
v_{a} & =Q_{a}(-d) \\
& =P(-d)+h_{a}(-d) \\
& =C_{a} d^{2} h(-d),
\end{aligned}
$$

thus $h(-d) \neq 0$ and

$$
C_{a}=\frac{v_{a}}{d^{2} h(-d)}
$$

So $a \mapsto C_{a}$ is analytic and non-constant with $C_{0}=0$. Let us summarize what we have: $Q_{a}(z)=P(z)+C_{a} z^{2} h(z)$ is a family of polynomials of degree $d+1, h(0) \neq 0$, with Siegel discs $\phi_{a}(S)$ for $|a| \leq \delta$. The family $\left(\phi_{a}\right)$ is a normalized family of 3quasiconformal maps, hence equicontinuous. Thus the conformal radii of the Siegel discs of $Q_{a}$ are bounded away from 0 for $|a| \leq \delta$. Applying Proposition 3.1 yields $\alpha \in \mathcal{B}$.

\subsection{The exponential family $E_{\lambda}$.}

Theorem 3.4. Let $\lambda=e^{2 \pi i \alpha}, \alpha \in \mathbb{R} \backslash \mathbb{Q}$. Then the function $E_{\lambda}(z)=\lambda z e^{z}$ is linearizable at 0 iff $\alpha \in \mathcal{B}$.

Proof. One direction, namely that $\alpha \in \mathcal{B}$ implies linearizability, is again due to the result of Brjuno and Rüssmann. So let us suppose that $E=E_{\lambda}$ is linearizable at 0, i.e. has a Siegel disc $S$ with $0 \in S$. Many ideas in the proof are very similar to those 
in the polynomial case. We will be a bit sketchy in these cases and concentrate on the differences.

Choose $\delta>0$ such that $\overline{D_{2 \delta}(0)} \subset S$. Then $E^{-1}\left(D_{2 \delta}(0)\right)=D_{1} \cup D_{2}$, where $D_{1}$ is the component contained in $S$ and $D_{2}$ is a domain contained in an unbounded Fatou component $S^{\prime}$. By choosing $\delta$ smaller we can arrange $D_{2} \subseteq\{z \in \mathbb{C} \mid \operatorname{Re} z<-2\}$. Again we choose a $C^{\infty}$-function $\eta:[0, \infty) \rightarrow[0,1]$ with $\eta(t)=0$ for $t \geq 4$ and $\eta(t)=1$ for $t \leq 1$. Set $A:=\left\{z \in S^{\prime}|| E(z) \mid \in[\delta, 2 \delta]\right\}$. Then define $E_{a}(z):=E(z)$ for $z \notin D_{2}$ and

$$
\begin{aligned}
E_{a}(z) & =E(z)+a \eta\left(\frac{|E(z)|^{2}}{\delta}\right) \\
& =E(z)+a \eta\left(\frac{E(z) \overline{E(z)}}{\delta}\right)
\end{aligned}
$$

for $z \in S^{\prime}$. Then both definitions coincide near $\partial S^{\prime}$ and $E_{a}$ is a $C^{\infty}$-function with partial derivatives

$$
\begin{aligned}
& \bar{\partial} E_{a}(z)=a \eta^{\prime}\left(\frac{|E(z)|^{2}}{\delta}\right) \frac{E(z)}{\delta} \\
& \partial E_{a}(z)=E^{\prime}(z)+a \eta^{\prime}\left(\frac{|E(z)|^{2}}{\delta}\right) \frac{\overline{E(z)}}{\delta}
\end{aligned}
$$

for $z \in S^{\prime}$. In $\mathbb{C} \backslash A$ the function $E_{a}$ is either $E$ or $E+a$, thus it is analytic there. For $z \in A$ we have $E^{\prime}(z)=\lambda(z+1) e^{z}=(1+1 / z) E(z)$ and $|z| \geq 2$, so $\left|E^{\prime}(z)\right| \geq|E(z)| / 2 \geq \delta / 2$. Setting $M:=\max \left|\eta^{\prime}\right|$, we obtain the estimates

$$
\begin{aligned}
\left|\bar{\partial} E_{a}(z)\right| & \leq 2 M|a|, \\
\left|\partial E_{a}(z)\right| & \geq \frac{\delta}{2}-2 M|a|
\end{aligned}
$$

for $z \in A$. Thus the dilatation of $E_{a}$ satisfies

$$
\left|\mu_{E_{a}}(z)\right| \leq \frac{2 M|a|}{\frac{\delta}{2}-2 M|a|}=: k_{a}
$$

and $k_{a} \rightarrow 0$ for $a \rightarrow 0$. Thus $E_{a}$ is $K_{a}$-quasiregular for $|a| \leq a_{0}$ with $K_{a}=$ $\left(1+k_{a}\right) /\left(1-k_{a}\right) \rightarrow 1$ for $a \rightarrow 0$. We will need this (small dilatation) in the exponential case, whereas in the polynomial case just quasiregularity was sufficient. Exactly the same pull-back method as in the polynomial case gives the $E_{a}$-invariant Beltrami coefficient

$$
\mu_{a}(z):= \begin{cases}\left(E^{n}\right)^{*} \mu_{E_{a}}(z) & \text { for } E^{n}(z) \in A, \\ 0 & \text { if no such } n \text { exists. }\end{cases}
$$

One easily sees $\left\|\mu_{a}\right\|=\left\|\mu_{E_{a}}\right\|$. We solve the Beltrami equation to get an analytic family of quasiconformal maps $\phi_{a}$ with complex dilatations $\mu_{a}$, normalized by $\phi_{a}(0)=0, \phi_{a}(-1)=-1$. Then $F_{a}:=\phi_{a} \circ E_{a} \circ \phi_{a}^{-1}$ is analytic, hence an entire function. The determination of $F_{a}$ is now the main difference to the polynomial case. Let us collect properties of $E_{a}$ : All the $E_{a}$ have Siegel discs $S$ centered at 0 with corresponding multiplier $\lambda$. They have one asymptotic value at $a$ and one critical point at -1 . (The modification did not create a new critical point but only moved the asymptotic value.)

The map $F_{a}$ inherits some of the properties of $E_{a}$ : As all $\phi_{a}$ are analytic in $S$, the $F_{a}$ have Siegel discs $\phi_{a}(S)$ centered at 0 and an asymptotic value at $v_{a}:=\phi_{a}(a)$. 
For $z$ near 0 (at least for $z \in S$ ), the function $\phi_{a}(z)$ is analytic in both variables, so $a \mapsto v_{a}$ is analytic. It is non-constant because $v_{0}=0$ and $v_{a} \neq 0$ for $a \neq 0$.

We will now determine the growth order of $F_{a}$. For a continuous function $f$ defined in a neighborhood of $\infty$ we define $M(r, f):=\max _{|z|=r}|f(z)|$ and the growth order $\gamma(f)$ by

$$
\gamma(f):=\limsup _{r \rightarrow \infty} \frac{\log ^{+} \log ^{+} M(r, f)}{\log r}
$$

where $\log ^{+} t=\log \max (1, t)$. (The growth order is usually denoted by $\rho$ but this will be reserved for the rotation number for circle homeomorphisms.) The growth order of $E$ is 1 , so the growth order of $E_{a}$ is 1 , too, because $\left|E-E_{a}\right| \leq a$. The maps $\phi_{a}$ and $\phi_{a}^{-1}$ are $K_{a}$-quasiconformal, so they are $1 / K_{a}$-Hölder continuous homeomorphisms of $\widehat{\mathbb{C}}$ fixing $\infty$. Maps of this type can only change the growth order by a factor of $K_{a}$ :

Lemma 3.5. Let $f$ be a continuous map in a neighborhood of $\infty$. Let $\phi: \mathbb{C} \rightarrow \mathbb{C}$ be a homeomorphism with $|\phi(z)| \leq C|z|^{K}$ for $|z| \geq r_{0}$. Then $\gamma(\phi f) \leq \gamma(f)$ and $\gamma(f \phi) \leq K \gamma(f)$.

Remark. In our applications the map $f$ will always be quasiregular and $\phi$ will be quasiconformal.

Proof. Let $\gamma=\gamma(f)$. For every $\varepsilon>0$ there exists $r=r(\varepsilon)$ with

$$
\log ^{+} \log ^{+}|f(z)| \leq(\gamma+\varepsilon) \log |z|
$$

for $|z| \geq r$, so

$$
|f(z)| \leq e^{|z|^{\gamma+\varepsilon}}
$$

for $|z| \geq r$. With the assumption on $\phi$ also

$$
|\phi f(z)| \leq C e^{K|z|^{\gamma+\varepsilon}}
$$

for $|z| \geq r$ and $|f(z)| \geq r_{0}$. Of course $\phi$ as a continuous function in $\mathbb{C}$ is bounded in $D_{r_{0}}(0)$, thus the points where $|f(z)|<r_{0}$ do not affect the growth order. The last inequality shows $\gamma(\phi f) \leq \gamma+\varepsilon$. This holds for arbitrary $\varepsilon>0$, so $\gamma(\phi f) \leq \gamma$.

Again using (1) and the assumption on $\phi$ we obtain

$$
\begin{aligned}
|f \phi(z)| & \leq e^{\left(C|z|^{K}\right)^{\gamma+\varepsilon}} \\
& =e^{C^{K}|z|^{K(\gamma+\varepsilon)}}
\end{aligned}
$$

for $|z| \geq r_{0}$ and $|\phi(z)| \geq r$. This shows that $\gamma(f \phi) \leq K(\gamma+\varepsilon)$ for every $\varepsilon>0$, so $\gamma(f \phi) \leq K \gamma$.

This lemma shows that the growth order $\gamma_{a}$ of $F_{a}$ satisfies $1 / K_{a} \leq \gamma_{a} \leq K_{a}$. We now choose $\delta>0$ so small that $K_{a}<2$ for $|a| \leq \delta$. Thus $1 / 2<\gamma_{a}<2$ for $|a| \leq \delta$. The derivative $F_{a}^{\prime}$ has the same growth order $\gamma_{a}$ and only one zero, namely at -1 . Such a function must be of the form $P_{a} e^{Q_{a}}$ where $P_{a}$ and $Q_{a}$ are polynomials. In our case, the growth order must be 1 and

$$
F_{a}^{\prime}(z)=\lambda(z+1) e^{b_{a} z}
$$


with some $b_{a} \neq 0$. Integrating this yields

$$
\begin{aligned}
F_{a}(z) & =\lambda \int_{0}^{z}(\zeta+1) e^{b_{a} \zeta} d \zeta \\
& =\frac{\lambda}{b_{a}}\left[\left(z+1-\frac{1}{b_{a}}\right) e^{b_{a} z}-1+\frac{1}{b_{a}}\right] .
\end{aligned}
$$

The asymptotic value is

$$
v_{a}=\lambda\left(\frac{1}{b_{a}^{2}}-\frac{1}{b_{a}}\right),
$$

thus

$$
\frac{1}{b_{a}}=\frac{1}{2}+\sqrt{\frac{1}{4}+\frac{v_{a}}{\lambda}},
$$

where the local branch of the square root with $\sqrt{1 / 4}=1 / 2$ is chosen. This shows that $b_{a}$ is an analytic non-constant function of $v_{a}$, thus analytic and non-constant in $a$, with $b_{0}=1$. We see that $F_{a}$ is an analytic family and so is the following linearly conjugate family:

$$
\begin{aligned}
G_{a}(z) & :=b_{a} F_{a}\left(\frac{z}{b_{a}}\right) \\
& =\lambda\left[\left(\frac{z}{b_{a}}+1-\frac{1}{b_{a}}\right) e^{z}-1+\frac{1}{b_{a}}\right] \\
& =\lambda z e^{z}+c_{a} z^{2} H(z)
\end{aligned}
$$

with $c_{a}=1 / b_{a}-1$ and

$$
\begin{aligned}
H(z) & =\lambda \frac{(z-1) e^{z}+1}{z^{2}} \\
& =\frac{\lambda}{2}+O(z) .
\end{aligned}
$$

These $G_{a}$ all have Siegel discs of uniform size and $c_{a}$ is analytic and non-constant with $c_{0}=0$. Thus $G_{a}$ satisfies the assumptions of Proposition 3.1 and we conclude $\alpha \in \mathcal{B}$.

\section{The Arnold family}

4.1. Circle diffeomorphisms. Let $F: \mathbb{T} \rightarrow \mathbb{T}$ be an orientation-preserving homeomorphism. Via the covering $E: \mathbb{R} \rightarrow \mathbb{T}, x \mapsto e^{2 \pi i x}$, it can be lifted to an increasing homeomorphism $f: \mathbb{R} \rightarrow \mathbb{R}$, satisfying $f(x+1)=f(x)+1$ for all $x \in \mathbb{R}$. The lift $f$ is unique up to an additive integer constant. We will mostly work with one fixed lift. For the lift $f$, the rotation number is defined as

$$
\rho(f):=\lim _{n \rightarrow \infty} \frac{f^{n}(x)-x}{n},
$$

where $f^{n}=f \circ f \circ \cdots \circ f$ is the $n$-th iterate of $f$. The limit exists uniformly in $x$ and is a real number. The rotation number of $F$ is defined as $\rho(F):=\rho(f) \bmod 1 \in \mathbb{R} / \mathbb{Z}$, which is independent of the choice of the lift. Poincaré showed that the rotation number is rational iff $F$ has a periodic orbit. Later Denjoy established that a $C^{2}$-diffeomorphism without periodic points is topologically conjugate to the rigid rotation $z \mapsto \lambda z$ with $\lambda=E(\rho(F))$, i.e. there exists an orientation-preserving homeomorphism $H: \mathbb{T} \rightarrow \mathbb{T}$ with $H(F(z))=\lambda H(z)$. If we normalize $H$ by 
$H(1)=1$, then $H$ is unique. The equivalent statement for the lift $f$ is the following: If $f: \mathbb{R} \rightarrow \mathbb{R}$ is an increasing $C^{2}$-diffeomorphism satisfying $f(x+1)=f(x)+1$ and if $\rho(f) \in \mathbb{R} \backslash \mathbb{Q}$, then there exists an increasing homeomorphism $h: \mathbb{R} \rightarrow \mathbb{R}$ with $h(x+1)=h(x)+1$ and

$$
h(f(x))=h(x)+\rho(f)
$$

for all $x \in \mathbb{R}$. (When speaking about lifts, we will call the map $x \mapsto x+\rho$ a rigid rotation.) Again the normalization $h(0)=0$ makes $h$ unique. In the sequel we restrict our attention to analytic circle diffeomorphism, to which Denjoy's theorem certainly applies. Arnold showed in $[\mathrm{Ar}]$ that $h$ need not even be absolutely continuous with respect to Lebesgue measure, despite the analyticity of $f$. In the same paper he proved that $h$ is analytic if one requires that the rotation number satisfies a certain Diophantine condition and if one additionally assumes that $f$ is close to a rigid rotation. Arnold's condition on the rotation number was already satisfied almost surely with respect to Lebesgue measure. His result on analytic linearizability was subsequently improved by Rüssmann, Herman and Yoccoz. Yoccoz found the sharp conditions on the rotation number for both local (i.e. assuming proximity to a rotation) and global statements [Yo2.

Global Conjugacy Theorem (Yoccoz). If $\rho \in \mathcal{H}$, then every analytic circle diffeomorphism with rotation number $\rho$ is analytically linearizable. If $\rho \notin \mathcal{H}$, then there exists an analytic circle diffeomorphism with rotation number $\rho$ which is not analytically linearizable.

Remark. The exact definition of $\mathcal{H}$ can be found in [PM1]. The set $\mathcal{H} \subsetneq \mathcal{B}$ has full (Lebesgue) measure and is invariant under $\rho \mapsto \rho+1$ and $\rho \mapsto-1 / \rho$.

Local Conjugacy Theorem (Yoccoz). If $\rho \in \mathcal{B}$ then there exists $R=R(\rho)$ such that any analytic circle diffeomorphism which extends univalently to the annulus $A_{R}=\{z \in \mathbb{C}|1 / R<| z \mid<R\}$ is analytically linearizable. The condition $\mathcal{B}$ is optimal.

Remark. We will also prove the optimality of $\mathcal{B}$ in the following. Yoccoz showed that analytic linearizability of $B_{t, a}(z)=e^{2 \pi i t} z^{2}(z+a) /(1+a z)$ for $a>3$ implies linearizability of $P(z)=\lambda z+z^{2}$, where $\lambda=e^{2 \pi i \rho\left(B_{t, a}\right)}$ and this implies $\rho\left(B_{t, a}\right) \in \mathcal{B}$. For any $R$ one can choose $a=a(R)>3$ large enough such that the function $B_{t, a}$ is univalent in $A_{R}$. Note that the condition of univalency in a large annulus implies proximity to the rigid rotation by classical distortion theorems.

Like the quadratic family is a prototype for linearizability of irrationally indifferent fixed points, the family $B_{t, a}$ is a prototype for the local linearization theorem for circle diffeomorphisms. Our purpose in the following is to show that the Arnold family $f_{a, b}: x \mapsto x+a+b \sin (2 \pi x), a \in \mathbb{R}, b \in(0,1 /(2 \pi))$, which is the lift of a family of analytic circle diffeomorphisms $F_{a, b}$, is also a prototype for the local linearization theorem. Still an open question is to determine a prototype for the global theorem. The corresponding conjecture about the Arnold family is: If $\rho \notin \mathcal{H}$, then there exist $a \in \mathbb{R}, b \in(0,1 /(2 \pi))$ such that $\rho\left(f_{a, b}\right)=\rho$ and $f_{a, b}$ is not analytically linearizable.

4.2. Linearizability in the Arnold family. From the real analytic point of view, the Arnold family is the simplest family of non-linear analytic circle diffeomorphisms, having only one non-linear Fourier coefficient. Its lift to the real line is 
defined by

$$
f_{a, b}(x):=x+a+b \sin (2 \pi x), \quad a, b \in \mathbb{R}, 0<b<1 /(2 \pi),
$$

which is a family of increasing analytic diffeomorphisms of $\mathbb{R}$, satisfying $f(x+1)=$ $f(x)+1$ for all $x \in \mathbb{R}$. Thus it projects to a family of analytic circle diffeomorphisms

$$
\begin{aligned}
F_{a, b}(z) & =E \circ f_{a, b} \circ E^{-1}(z) \\
& =e^{2 \pi i a} z e^{\pi b(z-1 / z)} .
\end{aligned}
$$

These maps extend to analytic self-maps of $\mathbb{C}^{*}$. They were investigated from the complex dynamical viewpoint in [Fa1] and [Fa2, also for other values of the parameters $a$ and $b$. We will identify $F_{a, b}$ with its extension to $\mathbb{C}^{*}$. When we want to stress that we speak about the extension we will call it the complex Arnold family.

Theorem 4.1. If $F_{a, b}$ is an analytically linearizable element of the Arnold family for some $a, b \in \mathbb{R}$ and $0<b<1 /(2 \pi)$, then $\rho\left(F_{a, b}\right) \in \mathcal{B}$. Conversely, if $\rho \in \mathcal{B}$, then there exist $a, b \in \mathbb{R}, 0<b<1 /(2 \pi)$ such that $\rho\left(F_{a, b}\right)=\rho$ and $F_{a, b}$ is analytically linearizable.

Remark. The second part of the theorem follows also from Yoccoz' local linearization theorem. However, we will give an independent proof of it for the Arnold family. The same result holds for the family $B_{a, b, d}(z)=e^{2 \pi i a} z^{d+1}[(z+b) /(1+b z)]^{d}$ for $a, b \in \mathbb{R}, d \in \mathbb{N}, b>2 d+1$. Instead of using $E_{\lambda}$ as a tool, one has to take $P_{\lambda, d}$ in that case. The proof is quite similar and becomes a bit easier there.

Let us sketch the proof of the theorem: We start by assuming analytic linearizability of $F_{a, b}$, so we have an Arnold-Herman ring containing $\mathbb{T}$ as an invariant curve. Then we quasiconformally paste a rotation inside $\mathbb{D}$ and show that the resulting map is quasiconformally conjugate to $E_{\lambda}(z)=\lambda z e^{z}$. The rotation number does not change under topological conjugation and we obtain $\lambda=e^{2 \pi i \rho\left(F_{a, b}\right)}$. This map has a Siegel disc instead of the original Arnold-Herman ring and thus by Theorem 3.4 we conclude $\rho\left(F_{a, b}\right) \in \mathcal{B}$. The other statement in the theorem is proved by reversing this procedure. Start with $E_{\lambda}$ where $\lambda=e^{2 \pi i \rho}$. By Brjuno's result $E_{\lambda}$ has a Siegel disc. We conjugate $E_{\lambda}$ by reflection in the unit circle and paste the dynamics of $E_{\lambda}$ with its conjugate in order to obtain a self-map of $\mathbb{C}^{*}$ having an Arnold-Herman ring. Finally we show that this map is quasiconformally conjugate to an element of the Arnold family. The idea of converting Arnold-Herman rings into Siegel discs and vice versa originated in Shishikura's paper $\underline{\mathrm{Sh}}$.

Proof. Let us assume that $F=F_{a, b}$ is analytically linearizable for some $a \in \mathbb{R}$ and $0<b<1 /(2 \pi)$. Let $\rho \in \mathbb{R} / \mathbb{Z}$ denote the rotation number of $F$ and define $\lambda:=E(\rho) \in \mathbb{T}$. For the rest of the proof we fix $a$ and $b$ and suppress them in our notation. Reflection in the unit circle will be denoted by $\tau(z)=1 / \bar{z}$.

Analytic linearizability implies that $F$ has an Arnold-Herman $\operatorname{ring} A \supseteq \mathbb{T}$, i.e. a maximal doubly connected rotation domain. There exist $R>1$ and a conformal isomorphism $\varphi: A \rightarrow A_{R}$ where $A_{R}=\{w \in \mathbb{C}|1 / R<| w \mid<R\}$ is an annulus. We normalize $\varphi$ to map the interior boundary component of $A$ onto the interior boundary component of $A_{R}$ and satisfy $\varphi(1)=1$. The map $F$ commutes with $\tau$, so $\tau(A)$ is also an Arnold-Herman ring containing $\mathbb{T}$ and this implies $A=\tau(A)$. Then $\tilde{\varphi}=\tau \varphi \tau$ is also a conformal isomorphism of $A$ onto $A_{R}$ subject to the same normalization, thus $\varphi \tau=\tau \varphi$. Also $\varphi$ conjugates $f$ to the rigid rotation $w \mapsto e^{2 \pi i \rho} w$ where $\rho \in \mathbb{R} \backslash \mathbb{Q}$ is the rotation number of $f$. 
Let $r:=(1+R) / 2$ and $A^{\prime}:=\varphi^{-1}\left(A_{r}\right)$. We construct a new map $\psi: A^{\prime} \cup \operatorname{int} A^{\prime} \rightarrow$ $D_{r}(0)$ which coincides with $\varphi$ in $A^{\prime}$ and is quasiconformal with $\psi(0)=0$. Of course $\psi$ will not be holomorphic. The possibility to construct such a quasiconformal extension of a conformal map between sets with analytic boundaries follows from the following lemma.

Lemma 4.2. Let $A$ and $B$ be doubly connected domains bounded by analytic curves. Let $\phi: \partial A \rightarrow \partial B$ be an orientation-preserving real-analytic diffeomorphism mapping the interior boundary curve of $A$ to the interior boundary curve of $B$. Then $\phi$ can be extended to a quasiconformal map $\hat{\phi}: A \rightarrow B$ with boundary values $\phi$.

Proof. One can find universal coverings from horizontal strips $S_{A}$ and $S_{B}$ to $A$ and $B$, respectively, with deck transformation groups generated by $z \mapsto z+1$. Then $\phi$ lifts to a map $\Phi: \partial S_{A} \rightarrow \partial S_{B}$ commuting with $z \mapsto z+1$. We extend $\Phi$ to $S_{A}$ by linear interpolation and get a quasiconformal map $\hat{\Phi}: S_{A} \rightarrow S_{B}$ with $\hat{\Phi}(z+1)=\Phi(z)+1$. This map projects to the desired extension of $\phi$. (A more detailed proof can be found in [Kr, Lemma 3.10].)

Let $\psi$ be a quasiconformal map in $A^{\prime} \cup \operatorname{int} A^{\prime}$ which coincides with $\varphi$ in $A^{\prime}$ and is the identity on a small disc about 0 . We define a new map $G: \mathbb{C} \rightarrow \mathbb{C}$ as follows:

$$
G(z):= \begin{cases}F(z) & \text { if }|z| \geq 1 \\ \psi^{-1} R_{\rho} \psi(z) & \text { if }|z|<1\end{cases}
$$

where $R_{\rho}(w)=e^{2 \pi i \rho} w$. Then $G$ is quasiconformal in $\mathbb{D}$ and analytic in a neighborhood of $\mathbb{C} \backslash \mathbb{D}$ because $G(z)=F(z)$ for all $z \in A_{r}$. Thus $G$ is quasiregular in $\mathbb{C}$, analytic outside $\mathbb{D}$ and has a rotation domain about the fixed point 0 . The rotation number of $\left.G\right|_{\mathbb{T}}$ is $\rho$. Let $K$ denote the dilatation of $\psi$. Then $G^{n}$ is $K^{2}$-quasiregular for all $n$ and the generalized qc-lemma gives a quasiconformal map $h: \mathbb{C} \rightarrow \mathbb{C}$ such that $H:=h G h^{-1}$ is analytic.

The map $H: \mathbb{C} \rightarrow \mathbb{C}$ is an entire function with $H(0)=0$ and a Siegel disc about 0 . The corresponding rotation number is $\rho$, so $H^{\prime}(0)=e^{2 \pi i \rho}$. Our goal is to show that $H(z)=\lambda z e^{b z}$ with some $b \in \mathbb{C}^{*}$. Then Theorem 3.4 gives $\rho \in \mathcal{B}$ because $b H(z / b)=E_{\lambda}(z)$.

By Lemma 3.5 the map $H$ has finite growth order. By construction $H$ does not have any zeros except at 0 , so 0 is an exceptional value. Thus $H(z)=\lambda z e^{P(z)}$ with a polynomial $P$ with $P(0)=0$. Let $d:=\operatorname{deg} P$. Then there are $d$ directions where $\operatorname{Re} P(z) \rightarrow+\infty$ and $\operatorname{Im} P(z)$ is bounded for $z \rightarrow \infty$ along these directions, separated by $d$ directions where $\operatorname{Re} P(z) \rightarrow-\infty$ and $\operatorname{Im} P(z)$ bounded. Thus there are $d$ directions where $H(z) \rightarrow \infty$ separated by $d$ directions where $H(z) \rightarrow 0$. This behavior is invariant under topological conjugation. The map $G$ coincides with $F$ outside $\mathbb{D}$ and $F$ has only one direction (along the positive real axis) where $F(z) \rightarrow \infty$ and one (the negative real axis) where $F(z) \rightarrow 0$. So we conclude $d=1$ and $H(z)=\lambda z e^{b z}$ as claimed. This finishes the first part of the proof.

Now let us take any $\rho \in \mathcal{B}$ and define $\lambda=e^{2 \pi i \rho}$. Our goal is to find an element of the Arnold family with rotation number $\rho$ which is analytically linearizable. By Brjuno's result the map $E_{\lambda}(z)=\lambda z e^{z}$ has a Siegel disc centered at 0 . Let $\gamma$ be an invariant analytic curve in the Siegel disc and choose $b>0$ with $2 / b<\operatorname{dist}(\gamma, 0)$. Define $\tilde{E}(z):=b E_{\lambda}(z / b)$. Then $\tilde{E}$ is an entire map with a Siegel disc $S \supset \mathbb{D}_{2}(0)$ about 0. Moreover, $\tilde{\gamma}:=b \gamma \subset \mathbb{C} \backslash D_{2}(0)$. Let $\phi: S \rightarrow D_{R}(0)$ be a conformal map with $\phi(0)=0$ and $\phi(\tilde{\gamma})=\{w \in \mathbb{C}|| w \mid=2\}$. Then $\phi F(z)=\lambda \phi(z)$ for $z \in S$. 
Define $\tilde{\phi}(z):=\phi(z)$ for $z \in \operatorname{ext} \tilde{\gamma}, \tilde{\phi}(z):=z$ for $z \in \mathbb{T}$. By Lemma 4.2 we can extend $\tilde{\phi}$ to a quasiconformal map defined in the domain bounded by $\tilde{\gamma}$ and $\mathbb{T}$. Let $\tilde{\phi}$ denote such a quasiconformal extension. Define

$$
G(z):= \begin{cases}\tilde{E}(z) & \text { for } z \in \operatorname{ext} \tilde{\gamma} \\ \tilde{\phi}^{-1}(\lambda \tilde{\phi}(z)) & \text { for } z \in \operatorname{int} \tilde{\gamma},|z| \geq 1 .\end{cases}
$$

Analytic curves are removable sets for quasiregular maps, so $G(z)$ is quasiregular in $\mathbb{C} \backslash \overline{\mathbb{D}}$ with $G(\mathbb{T})=\mathbb{T}$. By reflection it may be extended to a quasiregular self-map of $\mathbb{C}^{*}$. The domain $A$ where $G$ is not analytic is bounded by $\tilde{\gamma}$ and $\tau(\tilde{\gamma})$. There the map is quasiconformally conjugate to the rotation via $\tilde{\phi}$, which also extends by reflection. Thus again $G$ satisfies the assumptions of the qc-lemma and we can define an invariant Beltrami coefficient. Let

$$
\mu(z):= \begin{cases}\mu_{\tilde{\phi}}(z) & \text { for } z \in A, \\ \left(G^{n}\right)^{*} \mu_{\tilde{\phi}}(z) & \text { for } z \in G^{-n}(A) \backslash G^{-n+1}(A), \\ 0 & \text { otherwise. }\end{cases}
$$

The map $G$ commutes with $\tau$ and this implies $\tau \mu=\mu$. Let $\Phi: \mathbb{C} \rightarrow \mathbb{C}$ be a quasiconformal map with $\Phi(0)=0, \Phi(1)=1$ and $\mu_{\Phi}=\mu$ a.e. Then $\tau \Phi \tau$ is a map satisfying the same conditions and by the uniqueness part of the Measurable Riemann Mapping Theorem $\tau \Phi \tau=\Phi$, thus $\Phi$ commutes with $\tau$, too. In particular, $\Phi(\mathbb{T})=\mathbb{T}$.

Define $F:=\Phi G \Phi^{-1}$. Then $F$ is an analytic self-map of $\mathbb{C}^{*}$ commuting with $\tau$. By construction $F(\mathbb{T})=\mathbb{T}$ and $F$ is quasiconformally conjugate to a rotation in $\Phi(A) \supset \mathbb{T}$. This implies that $\left.F\right|_{\mathbb{T}}$ is analytically linearizable. The rotation number of $\left.F\right|_{\mathbb{T}}$ is $\rho$ as it does not change under topological conjugation. As an analytic self-map of $\mathbb{C}^{*}$, the map $F$ can be written uniquely as $F(z)=\zeta z^{m} e^{P(z)+Q(1 / z)}$ where $\zeta \in \mathbb{C}^{*}, m \in \mathbb{Z}$ and $P$ and $Q$ are entire functions with $P(0)=0=Q(0)$. Writing out the symmetry condition $F \tau(z)=\tau F(z)$ we obtain

$$
\zeta \bar{z}^{-m} e^{P(1 / \bar{z})+Q(\bar{z})}=\bar{\zeta}^{-1} \bar{z}^{-m} e^{-\bar{P}(z)-\bar{Q}(1 / z)}
$$

thus by uniqueness of this representation $\zeta \in \mathbb{T}$ and $Q(z)=-\bar{P}(\bar{z})$. The map $\left.F\right|_{\mathbb{T}}$ is an orientation-preserving analytic circle diffeomorphism, so $m=1$. The map $G$ has growth order 1 at infinity, thus $F$ has still finite growth order by Lemma 3.5. This implies that $P$ (and thus $Q$ ) is a polynomial of some degree $d \geq 1$. Exactly the same argument as in the first part of the proof shows that there are $d$ directions along which $F(z) \rightarrow \infty$ separated by $d$ directions where $F(z) \rightarrow 0$ for $z \rightarrow \infty$. This behavior is invariant under topological conjugation and the map $G$ has only one direction where $G(z) \rightarrow \infty$ and one where $G(z) \rightarrow 0$ for $z \rightarrow \infty$, thus $d=1$. So $F(z)=\zeta z e^{\omega z-\bar{\omega} / z}$ for some $\zeta \in \mathbb{T}$ and $\omega \in \mathbb{C}^{*}$. Conjugating $F$ by $S(z)=(|\omega| / \bar{\omega}) z$ we obtain

$$
\tilde{F}(z)=S F S^{-1}(z)=\zeta z e^{|\omega|(z-1 / z)} .
$$

This map $\tilde{F}$ is an element of the Arnold family because $\zeta \in \mathbb{T}, b:=|\omega| / \pi>0$ and $F$ has no critical points on $\mathbb{T}$, so $b<1 /(2 \pi)$. By construction it has an Arnold-Herman ring containing $\mathbb{T}$, so the theorem is proved. 
4.3. The complex Arnold family and its stable domains. For an analytic map $F: \mathbb{C}^{*} \rightarrow \mathbb{C}^{*}$ we define the Fatou set $\mathcal{F}(F)$ as the set of points where the family of iterates $\left(F^{n}\right)$ is equicontinuous. The Julia set is just the complement $J(F)=\mathbb{C}^{*} \backslash \mathcal{F}(F)$.

Theorem 4.3. Let $F_{a, b}$ be an element of the complex Arnold family with $a, b \in \mathbb{R}$, $0<b<1 /(2 \pi)$ and $\rho\left(F_{a, b}\right) \notin \mathbb{Q} / \mathbb{Z}$. If $F_{a, b}$ is not analytically linearizable, then $J\left(F_{a, b}\right)=\mathbb{C}^{*}$.

This is a complex dynamical theorem, motivated by N. Fagella's investigations of the complex Arnold family. In fact, we will show more:

Theorem 4.4. Let $a \in \mathbb{R}, 0<b<1 /(2 \pi)$ and $F_{a, b}(z)=e^{2 \pi i a} z e^{\pi b(z-1 / z)}$. Then the Fatou set $\mathcal{F}\left(F_{a, b}\right)$ consists entirely of periodic and preperiodic components. There is at most one cycle of periodic Fatou components. If $U_{1}, \ldots, U_{n}$ is such a cycle, then exactly one of the following holds:

1. The domains $U_{1}, \ldots, U_{n}$ are basins of attraction to an attracting cycle $\left\{z_{1}, \ldots\right.$, $\left.z_{n}\right\} \subset \mathbb{T}$ with $z_{j} \in U_{j}$

2. The domains $U_{1} \ldots, U_{n}$ are basins of attraction to a parabolic cycle $\left\{z_{1}, \ldots\right.$, $\left.z_{n}\right\} \subset \mathbb{T}$ with $z_{j} \in \partial U_{j}$.

3. The domain $U=U_{1}$ is an Arnold-Herman ring containing $\mathbb{T}$ and $F_{a, b}(U)=$ $U$.

Remark. Obviously this theorem implies the previous one. In the first two cases the domains $U_{j}$ are simply connected and $\bigcup_{j} \overline{U_{j}} \supset \mathbb{T}$. The first case occurs iff $F_{a, b}$ has two periodic cycles on $\mathbb{T}$, the second one iff it has one periodic cycle on $\mathbb{T}$. If there is no periodic orbit of $F_{a, b}$ on $\mathbb{T}$, either the third case holds or the Fatou set of $F_{a, b}$ is empty.

Theorem 4.4 is proved by combining some results of complex dynamics with the following idea: An invariant analytic curve in the Julia set on which the function is injective must attract at least one critical point. The proof of this statement is similar to the proof that boundaries of Siegel discs and Arnold-Herman rings attract critical points. In the complex Arnold family we have two critical points but their orbits are symmetric with respect to the unit circle. This already rules out the existence of attracting or parabolic cycles in $\mathbb{C}^{*} \backslash \mathbb{T}$. It turns out that the hardest part of the proof is to show that there can be no cycle of Siegel discs for the Arnold family. This is proved by a careful modification of Shishikura's ideas of turning indifferent periodic points into attractive ones [Sh. The argument additionally shows that there are no Cremer points, i.e. all periodic cycles outside $\mathbb{T}$ are repelling.

Proof. We will split up the proof in several steps, first ruling out the easy cases. The maps $F_{a, b}$ have no wandering domains and no Baker domains because they are self-maps of $\mathbb{C}^{*}$ with finitely many singular values. (See $[\mathrm{Ko}$ and $\mathrm{Ma}$.) The critical points are the two zeroes of $F_{a, b}^{\prime}(z)=e^{2 \pi i a}(1+\pi b(z+1 / z))$. The asymptotic values 0 and $\infty$ are not in the domain of definition.

Let us start with a simple observation; see also [Ba]. Recall that $\tau(z)=1 / \bar{z}$ denotes reflection in $\mathbb{T}$.

Lemma 4.5. If $F_{a, b}$ has an Arnold-Herman ring $A$, then $\mathbb{T} \subset A$ and $\tau(A)=A$. 
Proof. Assume that there is an Arnold-Herman $A$ ring of period $n \geq 1$ of $F_{a, b}$. Then there exists an analytic curve $\gamma \subset A$ with $F_{a, b}^{n}(\gamma)=\gamma$. Assume first that $0 \notin \operatorname{int} \gamma$. This implies that $F_{a, b}^{n}(\operatorname{int} \gamma)=\operatorname{int} \gamma$, so $F_{a, b}^{n}$ has a fixed point in int $\gamma$ and $\gamma$ is contained in a Siegel disc, contradicting our assumption. So let us assume that $0 \in$ int $\gamma$. If $\gamma \cap \mathbb{T} \neq \emptyset$, then $\gamma=\mathbb{T}$ because $F_{a, b}(\mathbb{T})=\mathbb{T}$ and an invariant curve in an Arnold-Herman ring is determined by one of its points. Otherwise, $\gamma \subset \mathbb{D}$ or $\gamma \subset \mathbb{C} \backslash \overline{\mathbb{D}}$. In either case let $G$ be the domain bounded by $\mathbb{T}$ and $\gamma$. As in the first case we obtain $F_{a, b}^{n}(G)=G$ and so $G$ is contained in an Arnold-Herman ring. This implies that $\gamma$ and $\mathbb{T}$ belong to the same Arnold-Herman ring. The second assertion of the lemma follows from $\tau \circ F_{a, b}=F_{a, b} \circ \tau$, implying that $\tau(A)$ is a component of the Fatou set whenever $A$ is.

The next lemma is not difficult to prove but is one of the key ingredients. It shows that the unit circle "needs" a critical point in any case. The set $P(F):=$ $\overline{\left\{F^{n}(c) \mid n \geq 1, F^{\prime}(c)=0\right\}}$ is the post-critical set of $F$. (In general we would have to take asymptotic values into account, too. The Arnold family has no asymptotic values in $\mathbb{C}^{*}$, so we may use this simplified definition.)

Lemma 4.6. If $\mathbb{T} \subset J\left(F_{a, b}\right)$, then $\mathbb{T} \subset P\left(F_{a, b}\right)$.

Proof. First observe that the condition $\mathbb{T} \subseteq J\left(F_{a, b}\right)$ implies $\rho\left(F_{a, b}\right) \notin \mathbb{Q}$ because otherwise all points on $\mathbb{T}$ converge towards a finite number of periodic cycles on $\mathbb{T}$, so the unit circle intersects basins of attraction to a parabolic or attracting cycle. Let us assume that the claim is wrong. Then there exists $z_{0} \in \mathbb{T}$ and $\varepsilon>0$ such that $D_{\varepsilon}\left(z_{0}\right) \cap P\left(F_{a, b}\right)=\emptyset$. So all branches of $F_{a, b}^{-n}$ exist in $D_{\varepsilon}\left(z_{0}\right)$. Let $G_{n}: D_{\varepsilon}\left(z_{0}\right) \rightarrow \mathbb{C}^{*}$ denote the branch of $F^{-n}$ with $z_{n}=G_{n}\left(z_{0}\right) \in \mathbb{T}$. By Denjoy's theorem there exists a homeomorphism $H: \mathbb{T} \rightarrow \mathbb{T}$ with $H\left(F_{a, b}(z)\right)=\lambda H(z)$, where $\lambda=e^{2 \pi i \rho\left(F_{a, b}\right)}$. Now choose a sequence $\left(n_{k}\right)$ such that $\lambda^{n_{k}} \rightarrow 1$ for $k \rightarrow \infty$. The sequence $\left(G_{n}\right)_{n \in \mathbb{N}}$ is a normal family because it does not assume the values $0, \infty, c_{1}$ and $c_{2}$, where $c_{j}$ are the critical points of $F_{a, b}$. So we may assume that the sequence $\left(n_{k}\right)$ is already chosen to satisfy $G_{n_{k}} \rightarrow G$ locally uniformly for $k \rightarrow \infty$. The functional equation gives $H\left(G_{n_{k}}(z)\right)=\lambda^{-n_{k}} H(z)$, so in the limit $H(G(z))=H(z)$ for all $z \in \mathbb{T} \cap D_{\varepsilon}\left(z_{0}\right)$, implying $G(z)=z$ for all $z \in D_{\varepsilon}\left(z_{0}\right)$. Then also the inverse functions $F^{n_{k}}$ are univalent and converge to the identity locally uniformly in $D_{\varepsilon}\left(z_{0}\right)$, contradicting the fact $D_{\varepsilon}\left(z_{0}\right) \cap J\left(F_{a, b}\right) \neq \emptyset$. (Univalency suffices because this first shows that all iterates $F^{n}$ are univalent and do not assume the value 0 . The Koebe 1/4-Theorem then gives $\left|\left(F^{n}\right)^{\prime}\left(z_{0}\right)\right| \leq 4 / \varepsilon$ and this implies normality.)

Lemma 4.7. If $F_{a, b}$ has an attracting or parabolic cycle $Z$ then $Z \subset \mathbb{T}$.

Proof. This is a consequence of the preceding lemma and the fact that $F_{a, b}$ has only two critical points $c_{1}$ and $c_{2}$ which have symmetric orbits. If $\rho\left(F_{a, b}\right)$ is rational, then there is an attracting or parabolic cycle $Y \subset \mathbb{T}$ and $F_{a, b}^{n}\left(c_{j}\right)$ converges to $Y$ for $j=1$ or $j=2$. Due to symmetry $F_{a, b}^{n}\left(c_{j}\right)$ then converges to $Y$ for $j=1$ and $j=2$. Any other attracting or parabolic cycle would also have to attract a critical point, thus $Y=Z$ is the only attracting or parabolic cycle in this case. If $\rho\left(F_{a, b}\right)$ is irrational, then either $F_{a, b}$ has an Arnold-Herman $\operatorname{ring} A$ or $\mathbb{T} \subset J\left(F_{a, b}\right)$. In the first case $\partial A \subseteq P\left(F_{a, b}\right)$ and in the second case $\mathbb{T} \subseteq P\left(F_{a, b}\right)$. Assume that $F_{a, b}$ has an attracting cycle $Z$. Then one of the critical points converges to $Z$ under iteration of $F_{a, b}$, thus the orbit has a finite set of accumulation points. The other critical point has an orbit which is symmetric w.r.t. the unit circle, so $P\left(F_{a, b}\right)$ consists of 
the iterates $F^{n}\left(c_{j}\right)$ and a finite number of accumulation points. This contradicts the fact that $P\left(F_{a, b}\right)$ disconnects the plane and hence has to be uncountable.

Collecting everything up to here, the only claim which remains to be shown is the non-existence of Siegel discs for the Arnold family. This is the most difficult and technically rather involved part of the proof.

Proposition 4.8. The map $F_{a, b}$ does not have irrationally indifferent periodic points. In particular, all periodic points in $\mathbb{C}^{*} \backslash \mathbb{T}$ are repelling.

Proof. Define $T(z):=i(z-1) /(z+1)$ and $G_{a, b}(z):=T F_{a, b} T^{-1}(z)$. Then $G_{a, b}$ is an analytic self-map of $\hat{\mathbb{C}} \backslash\{i,-i\}$ with $G_{a, b}(\hat{\mathbb{R}})=\hat{\mathbb{R}}$, where $\hat{\mathbb{R}}=\mathbb{R} \cup\{\infty\}$. The proof proceeds by contradiction. Assume that $F_{a, b}$ has a cycle of irrationally indifferent periodic points $\left(z_{1}, \ldots, z_{n}\right)$. Then $\left(w_{1}, \ldots, w_{n}\right), w_{j}=T\left(z_{j}\right)$, is an irrationally indifferent cycle for $G_{a, b}$ with the same multiplier $\lambda:=\left(G_{a, b}^{n}\right)^{\prime}\left(w_{1}\right)$. Also $\left(\bar{w}_{1}, \ldots, \bar{w}_{n}\right)$ is an irrationally indifferent cycle for $G_{a, b}$ with multiplier $\bar{\lambda} \neq$ $\lambda$. In particular, the numbers $w_{1}, \ldots, w_{n}, \bar{w}_{1}, \ldots, \bar{w}_{n}$ are mutually distinct. As $F_{a, b}$ has no exceptional values in $\mathbb{C}^{*}$, the set $F_{a, b}^{-1}\left(z_{1}\right)$ is infinite. We choose $z_{0} \in$ $F_{a, b}^{-1}\left(z_{1}\right), z_{0} \neq z_{n}$ and set $w_{0}:=T\left(z_{0}\right)$. Of course also $G_{a, b}\left(w_{0}\right)=\left(w_{1}\right), w_{0} \neq w_{n}$ and $G_{a, b}\left(\bar{w}_{0}\right)=\bar{w}_{1}$. The following interpolation result will be the main technical tool to apply Shishikura's technique of making indifferent points attracting via quasiconformal surgery.

Lemma 4.9. There exists a rational map $R$ of degree $4 n+2$ with $R(\hat{\mathbb{R}}) \subset \mathbb{R}$, satisfying

1. $R^{-1}(\infty)=\left\{w_{0}, \bar{w}_{0}\right\}$;

2. $R\left(w_{k}\right)=0=R\left(\bar{w}_{k}\right)$ for $k=1, \ldots, n$;

3. $R^{\prime}\left(w_{k}\right)=-1=R^{\prime}\left(\bar{w}_{k}\right)$ for $k=1, \ldots, n$;

4. $R(i)=0=R(-i)$.

Proof. We construct $R$ in two steps. Write $R(w)=r(w) p(w)$ where

$$
r(w)=\frac{\left(w^{2}+1\right) \prod_{k=1}^{n}\left(w-w_{k}\right)\left(w-\bar{w}_{k}\right)}{\left(\left(w-w_{0}\right)\left(w-\bar{w}_{0}\right)\right)^{2 n+1}}
$$

and $p$ is a polynomial of degree at most $2 n-1$ with real coefficients satisfying $p\left(w_{k}\right)=-1 /\left(r^{\prime}\left(w_{k}\right)\right)$. (The numbers $w_{k}, \bar{w}_{k}$ are all simple zeros of $r$, so $r^{\prime}\left(w_{k}\right) \neq 0$.) The construction of $p$ will be carried out in the next lemma. Comparing the degrees of numerator and denominator, we see $R(\infty)=0$, so (1) is satisfied. Because $r$ and $p$ have real coefficients, $R(\hat{\mathbb{R}}) \subset \mathbb{R}$ holds. Conditions (2) and (4) are satisfied for $r$, so they hold for $R$, too. Condition (3) follows from $R^{\prime}\left(w_{k}\right)=r^{\prime}\left(w_{k}\right) p\left(w_{k}\right)+$ $r\left(w_{k}\right) p^{\prime}\left(w_{k}\right)=-1$, so $R$ has all the required properties.

Lemma 4.10. Given $n$ points $w_{1}, \ldots, w_{n}$, such that $w_{1}, \ldots, w_{n}, \bar{w}_{1}, \ldots, \bar{w}_{n}$ are mutually distinct and $n$ arbitrary complex numbers $c_{1}, \ldots, c_{n}$, there exists a unique polynomial $P$ of degree at most $2 n-1$ with real coefficients satisfying $P\left(w_{k}\right)=c_{k}$ and $P\left(\bar{w}_{k}\right)=\bar{c}_{k}$ for $k=1, \ldots, n$.

Proof. Uniqueness is easy: If $P$ and $\tilde{P}$ both solve the problem, then $P-\tilde{P}$ is a polynomial of degree $\leq 2 n-1$ with $2 n$ zeros, so $P-\tilde{P} \equiv 0$. Existence is proved similar to Lagrange interpolation. We construct polynomials $P_{1}, \ldots, P_{n}$ of degree 
$\leq 2 n-1$ with real coefficients such that $P_{k}\left(w_{j}\right)=0$ for $j \neq k$ and $P_{k}\left(w_{k}\right)=c_{k}$. Then $P:=\sum_{k=1}^{n} P_{k}$ is the required polynomial. Define

$$
p_{k}(w):=\prod_{\substack{j=1 \\ j \neq k}}^{n}\left(w-w_{j}\right)\left(w-\bar{w}_{j}\right) .
$$

Then $p_{k}$ is a polynomial of degree $2 n-2$ with real coefficients, $p_{k}\left(w_{j}\right)=0$ for $j \neq k$ and $d_{k}:=p_{k}\left(w_{k}\right) \neq 0$. Because $\left\{1, w_{k}\right\}$ is a base for $\mathbb{C}$ as a vector space over $\mathbb{R}$, there exist $a_{k}, b_{k} \in \mathbb{R}$ such that $a_{k} w_{k}+b_{k}=c_{k} / d_{k}$. Define $P_{k}(w):=\left(a_{k} w+b\right) p_{k}(w)$. Then $P_{k}\left(w_{j}\right)=0$ for $j \neq k$ and $P_{k}\left(w_{k}\right)=c_{k}$. Moreover, $P_{k}$ is a polynomial of degree $\leq 2 n-1$ with real coefficients, which additionally implies $P\left(\bar{w}_{k}\right)=\bar{c}_{k}$.

We proceed to construct the perturbation of $G=G_{a, b}$. Let $\eta:[0, \infty) \rightarrow[0, \infty)$ be a $C^{\infty}$-function with $\eta(t)=0$ for $t \leq 1$ and $\eta(t)=1$ for $t \geq 2$. Define

$$
H_{\varepsilon}(w):=w+\varepsilon \eta\left(\varepsilon^{-1 /(2 n+2)} \max \left(\left|w-w_{0}\right|,\left|w-\bar{w}_{0}\right|\right)\right) R(w)
$$

with the previously constructed function $R$. For $\varepsilon \leq \varepsilon_{0}$ the map $H_{\varepsilon}$ is quasiconformal in $\mathbb{C}$ and analytic outside $\overline{D_{2 \varepsilon^{1 /(2 n+2)}}\left(w_{0}\right)} \cup \overline{D_{2 \varepsilon^{1 /(2 n+2)}}\left(\bar{w}_{0}\right)}$. Furthermore, $H_{\varepsilon}(i)=i, H_{\varepsilon}(-i)=-i, H_{\varepsilon}(\mathbb{R})=\mathbb{R}$ and $H_{\varepsilon}$ commutes with complex conjugation. Define $G_{\varepsilon}:=G \circ H_{\varepsilon}$. Then for $\varepsilon \leq \varepsilon_{0}$ the map $G_{\varepsilon}$ is a quasiregular self-map of $\hat{\mathbb{C}} \backslash\{i,-i\}$, commuting with complex conjugation and satisfying $G_{\varepsilon}\left(w_{k}\right)=w_{k+1}$, $G_{\varepsilon}\left(w_{n}\right)=w_{1},\left(G_{\varepsilon}^{n}\right)^{\prime}\left(w_{1}\right)=(1-\varepsilon)^{n} \lambda$. So $\left\{w_{1}, \ldots, w_{n}\right\}$ is an attracting cycle of $G_{\varepsilon}$ and our aim is to show that $G_{\varepsilon}\left(\overline{D_{2 \varepsilon^{1 /(2 n+2)}}\left(w_{0}\right)}\right)$ lies in the basin of attraction of this cycle for sufficiently small $\varepsilon$.

There exists an analytic map $\varphi(z)=z+O\left(z^{2}\right)$, defined in a neighborhood of 0 , satisfying

$$
\varphi G^{n} \varphi^{-1}(w)=\lambda w+O\left(w^{2 n+5}\right) .
$$

(See $\mathrm{Be}$ Thm. 6.10.5], the rest of the proof also closely follows Beardon's exposition of Shishikura's result.) Consider the function

$$
\Phi(\varepsilon, w):=\varphi G_{\varepsilon}^{n} \varphi^{-1}(w)
$$

for $w$ and $\varepsilon$ near 0 . This function is separately analytic in $\varepsilon$ and $w$ and continuous 1 So it can be represented by a double power series

$$
\Phi(\varepsilon, w)=\sum_{k=0}^{\infty} \sum_{l=0}^{\infty} a_{k, l} w^{k} \varepsilon^{l} .
$$

For $\varepsilon=0$ we obtain

$$
\lambda w+O\left(w^{2 n+5}\right)=\Phi(0, w)=\sum_{k=0}^{\infty} a_{k, 0} w^{k}
$$

and for $w=0$ similarly

$$
0=\Phi(\varepsilon, 0)=\sum_{l=0}^{\infty} a_{0, l} \varepsilon^{l}
$$

Furthermore, we know the $w$-derivative at $(\varepsilon, 0)$, so

$$
(1-\varepsilon)^{n} \lambda=\frac{\partial \Phi}{\partial w}(\varepsilon, 0)=\sum_{l=0}^{\infty} a_{1, l} \varepsilon^{l} .
$$

\footnotetext{
${ }^{1}$ Continuity follows from Hartogs' theorem but it is easy to see directly in our case.
} 
Taking all this together and observing that $a_{0,0}$ and $a_{1,0}$ appear twice, we get

$$
\begin{aligned}
\varphi G_{\varepsilon}^{n} \varphi^{-1}(w) & =\Phi(\varepsilon, w) \\
& =\varphi G^{n} \varphi^{-1}(w)-\lambda w+(1-\varepsilon)^{n} \lambda w+\sum_{k=2}^{\infty} \sum_{l=1}^{\infty} a_{k, l} w^{k} \varepsilon^{l} \\
& =\lambda w\left[(1-\varepsilon)^{n}+O\left(w^{2 n+4}\right)+O(\varepsilon w)\right]
\end{aligned}
$$

for $\varepsilon$ and $w$ near 0 . If $\left|w-w_{0}\right|=2 \varepsilon^{1 /(2 n+2)}$, then

$$
\begin{aligned}
\left|G_{\varepsilon}(w)-w_{1}\right| & =\left|G(w)+\varepsilon R(w)-w_{1}\right| \\
& \leq c_{1}\left|w-w_{0}\right|+\varepsilon|R(w)| \\
& \leq 2 c_{1} \varepsilon^{1 /(2 n+2)}+\varepsilon \frac{c_{2}}{\left|w-w_{0}\right|^{2 n+1}} \\
& \leq 2 c_{1} \varepsilon^{1 /(2 n+2)}+c_{2} \varepsilon^{1-(2 n+1) /(2 n+2)} \\
& \leq c_{3} \varepsilon^{1 /(2 n+2)} .
\end{aligned}
$$

For $|w| \leq \varepsilon^{1 /(2 n+3)}$ we have

$$
\begin{aligned}
\left|\varphi G_{\varepsilon}^{n} \varphi^{-1}(w)\right| & \leq|w|\left[(1-\varepsilon)^{n}+O\left(\varepsilon^{(2 n+4) /(2 n+3)}\right)+O\left(\varepsilon^{1+1 /(2 n+3)}\right)\right] \\
& =|w|\left(1-n \varepsilon+O\left(\varepsilon^{1+1 /(2 n+3)}\right)\right) \\
& \leq|w|\left(1-\frac{n}{2} \varepsilon\right) \\
& <|w|
\end{aligned}
$$

for $\varepsilon<\varepsilon_{1}$. This shows that $\varphi^{-1}\left(\overline{D_{\varepsilon^{1 /(2 n+3)}}(0)}\right)$ is contained in the attractive basin of $w_{1}$. The derivative of $\varphi$ at 0 is 1 , so we may additionally assume $\varphi^{-1}\left(D_{\varepsilon^{1 /(2 n+3)}(0)}\right) \supseteq$ $D_{\varepsilon^{1 /(2 n+3) / 2}}\left(w_{1}\right)$. For small $\varepsilon$ also $c_{3} \varepsilon^{1 /(2 n+2)}<\varepsilon^{1 /(2 n+3)} / 2$, so $G_{\varepsilon}\left(D_{2 \varepsilon^{1 /(2 n+2)}}\left(w_{0}\right)\right)$ is contained in the attractive basin of $w_{1}$.

So finally the assumptions of the qc-lemma are satisfied. We repeat the construction of the invariant Beltrami coefficient in order to obtain the symmetry properties of the quasiconformal conjugation. Define $D:=D_{2 \varepsilon^{1 /(2 n+2)}}\left(w_{0}\right) \cup D_{2 \varepsilon^{1 /(2 n+2)}}\left(\bar{w}_{0}\right)$ and

$$
\mu(w):= \begin{cases}\mu_{G_{\varepsilon}^{k}}(w) & \text { for } G_{\varepsilon}^{k-1}(w) \in \bar{D} \\ 0 & \text { otherwise. }\end{cases}
$$

Then $\mu$ is $G_{\varepsilon}$-invariant and $\mu(\bar{w})=\mu(w)$ for all $w \in \mathbb{C}$. So there exists a quasiconformal homeomorphism $\psi: \mathbb{C} \rightarrow \mathbb{C}$, normalized by $\psi(i)=i, \psi(-i)=-i$ satisfying $\mu_{\psi}=\mu$ almost everywhere. Due to the symmetry of $\mu$, also $\tilde{\psi}(w):=\overline{\psi(\bar{w})}$ satisfies $\mu_{\tilde{\psi}}=\mu$ almost everywhere and $\tilde{\psi}(i)=i, \tilde{\psi}(-i)=-i$. By the uniqueness part of the Measurable Riemann Mapping Theorem, we get that $\tilde{\psi}=\psi$, so $\psi$ commutes with complex conjugation.

Define $\tilde{G}:=\psi G_{\varepsilon} \psi^{-1}$. This is an analytic self-map of $\hat{\mathbb{C}} \backslash\{i,-i\}$, thus $\tilde{F}:=$ $T^{-1} \tilde{G} T$ is an analytic self-map of $\mathbb{C}^{*}$, commuting with $\tau$. We want to show that $\tilde{F}$ has finite growth order at $\infty$. In order to do so, we first observe that $G_{\varepsilon}=G \circ H_{\varepsilon}$ with $H_{\varepsilon}(i+w)=i+H_{\varepsilon}^{\prime}(i) w+O\left(w^{2}\right)$ and $H_{\varepsilon}^{\prime}(i) \rightarrow 1$ for $\varepsilon \rightarrow 0$. So we may assume

$$
\frac{1}{2} \leq\left|\frac{H_{\varepsilon}(i+w)-i}{w}\right| \leq 2
$$


for $|w|$ and $\varepsilon$ small. Furthermore, $\tilde{G}=\psi G H_{\varepsilon} \psi^{-1}$ where $\psi$ and $\psi^{-1}$ are $1 / K$-Hölder continuous. So there exists a constant $C$ with

$$
\frac{1}{C}|w|^{K} \leq\left|H_{\varepsilon}\left(\psi^{-1}(i+w)\right)-i\right| \leq C|w|^{1 / K}
$$

for small $|w|$. Let $\tilde{M}(r)$ denote the maximal value of $1 /|\tilde{G}(w)-i|$ for $|w-i|=r$. Then the growth order of $\tilde{F}=T^{-1} \tilde{G} T$ at $\infty$ is

$$
\gamma(\tilde{F})=\limsup _{r \rightarrow 0} \frac{\log \log \tilde{M}(r)}{\log (1 / r)} .
$$

Let $M(r)$ denote the maximal value of $1 /|G(w)-i|$ for $|w-i|=r$. We know that

$$
\limsup _{r \rightarrow 0} \frac{\log \log M(r)}{\log (1 / r)}=1 \text {. }
$$

The same calculations as in Lemma 3.5 lead to

$$
\gamma(\tilde{F}) \leq K .
$$

Analytic self-maps of $\mathbb{C}^{*}$ can always uniquely be represented as

$$
\tilde{F}(z)=\lambda z^{m} e^{P(z)+Q(1 / z)}
$$

with $\lambda \in \mathbb{C}^{*}, m \in \mathbb{Z}$ and entire functions $P$ and $Q$ with $P(0)=Q(0)=0$. By symmetry

$$
\begin{aligned}
\tilde{F}(z) & =\tau \tilde{F} \tau(z) \\
& =\tau(\lambda) z^{m} e^{-\bar{P}(1 / \bar{z})-\bar{Q}(\bar{z})}
\end{aligned}
$$

so $Q(z)=-\bar{P}(\bar{z})$ and $|\lambda|=1$. Because $\tilde{F}$ has finite growth order at $\infty$, the map $P$ is a polynomial. The number $m$ is the winding number of $\tilde{F}$ w.r.t. 0 and may be evaluated as $m=1 /(2 \pi i) \int_{\mathbb{T}} \frac{F^{\prime}(z)}{F(z)} d z$. Because $\tilde{F}$ is still an orientation preserving circle diffeomorphism on $\mathbb{T}$, we get $m=1$. (The winding number cannot be changed by small perturbations.) The topological behavior at $\infty$ is determined by the degree $d$ of $P$. There are $d$ directions where the real part of $P$ tends to $+\infty$, separated by $d$ directions where it tends to $-\infty$. So the value of $\tilde{F}$ tends to $\infty$ and 0 , respectively, along these directions. This behavior is not changed by small perturbations and it is also unchanged under quasiconformal conjugacy. Thus $\operatorname{deg} P=1$ and $P(z)=\omega z$ with some $\omega \in \mathbb{C}^{*}$. So

$$
\tilde{F}(z)=\lambda z e^{\omega z-\bar{\omega} / z}
$$

with $\lambda \in \mathbb{T}$. Conjugation by $S(z):=(|\omega| / \bar{\omega}) z$ gives

$$
S \tilde{F} S^{-1}(z)=\lambda z e^{|\omega|(z-1 / z)} .
$$

This new map is an element of the Arnold family having an attracting cycle in $\mathbb{C}^{*} \backslash \mathbb{T}$, thus contradicting Lemma 4.7.

\section{Geometry of rotation domains}

In this section we are going to make use of the quasiconformal techniques to prove that the Siegel discs of $P_{\lambda, d}$ and $E_{\lambda}$ are quasidiscs with a critical point on the boundary if the rotation number is of "constant type". 
Definition 5.1. A number $\rho \in \mathbb{R} \backslash \mathbb{Q}$ if of constant type if

$$
\rho=a_{0}+\frac{1}{a_{1}+\frac{1}{a_{2}+\ddots} .}
$$

with $a_{0} \in \mathbb{Z}, a_{n} \in \mathbb{N}$ and $\sup _{n} a_{n}<\infty$.

Remark. This is equivalent to the existence of a constant $c>0$ with $|\rho-p / q| \leq c q^{-2}$ for all $p \in \mathbb{Z}, q \in \mathbb{N}$.

Definition 5.2. A homeomorphism $H: \mathbb{T} \rightarrow \mathbb{T}$ is quasisymmetric if it preserves orientation and there exists $M \in \mathbb{R}$ with

$$
\left|\frac{H(\zeta z)-H(z)}{H(z)-H\left(\zeta^{-1} z\right)}\right| \leq M
$$

for all $\zeta, z \in \mathbb{T}, \zeta \neq 1$.

An orientation-preserving homeomorphism $F: \mathbb{T} \rightarrow \mathbb{T}$ is quasisymmetrically linearizable if there exists a quasisymmetric homeomorphism $H: \mathbb{T} \rightarrow \mathbb{T}$ with $H(F(z))=\lambda H(z)$ with $\lambda=e^{2 \pi i \rho(F)}$.

The key ingredient is the following theorem $[\mathrm{He} 2, \underline{\mathrm{Sw}}$.

Theorem 5.3 (Herman, Świątek). If $F: \mathbb{T} \rightarrow \mathbb{T}$ is an analytic homeomorphism with $\rho(F)$ of constant type, then $F$ is quasisymmetrically linearizable.

This theorem is an easy consequence of Denjoy's result if $F$ has no critical points. The main point is that it still holds in the presence of critical points. Here the conjugation cannot be differentiable as can be seen from the functional equation.

Let $f_{a}(x):=x+a+(1 / 2 \pi) \sin (2 \pi x)$. This is an analytic homeomorphism of $\mathbb{R}$ with critical points in $1 / 2+n, n \in \mathbb{Z}$. The rotation number $\rho_{a}:=\rho\left(f_{a}\right)$ is a continuous non-decreasing function of $a$ with $\rho_{a+1}=\rho_{a}+1$, so it is surjective. The map $f_{a}$ projects to $F_{a}(z)=e^{2 \pi i a} z e^{(z-1 / z) / 2}$ on $\mathbb{T}$ which is an analytic circle of homeomorphisms with critical point -1 . For every $\rho \in \mathbb{R}$ there exists $a=a(\rho) \in \mathbb{R}$ with $\rho_{a(\rho)}=\rho$. Now it is not difficult to prove the following theorem.

Theorem 5.4. Let $\rho \in \mathbb{R} \backslash \mathbb{Q}$ be of constant type and $\lambda=e^{2 \pi i \rho}$. Then $E_{\lambda}(z)=\lambda z e^{z}$ has a Siegel disc $S$ where $\partial S$ is a quasicircle and $-1 \in \partial S$.

Proof. Choose $a=a(\rho)$ as above. Then $F=F_{a}$ is an analytic circle homeomorphism with $\rho(F)=\rho$ of constant type. So there exists a quasisymmetric homeomorphism $H: \mathbb{T} \rightarrow \mathbb{T}$ with $H(F(z))=\lambda H(z)$. By the theorem of Ahlfors and Beurling $H$ has a quasiconformal extension $\hat{H}: \overline{\mathbb{D}} \rightarrow \overline{\mathbb{D}}$. We may normalize $\hat{H}$ by $\hat{H}(0)=0$. Define

$$
\tilde{F}(z):= \begin{cases}F(z) & \text { if }|z| \geq 1 \\ \hat{H}^{-1}(\lambda \hat{H}(z)) & \text { if }|z| \leq 1 .\end{cases}
$$

By the functional equation on $\mathbb{T}$ this definition is unambiguous and $\tilde{F}$ is quasiregular in $\mathbb{C} \backslash \mathbb{T}$ and continuous in $\mathbb{C}$. Analytic curves are removable sets for quasiconformal maps, so $\tilde{F}$ is quasiregular in $\mathbb{C}$. The map $\tilde{F}$ satisfies the assumptions of the qclemma because it is quasiconformally conjugate to a rotation in $\overline{\mathbb{D}}$. Thus there exists a quasiconformal homeomorphism $\Phi: \mathbb{C} \rightarrow \mathbb{C}$, normalized by $\Phi(0)=0$, $\Phi(-1)=-1$ such that $E:=\Phi F \Phi^{-1}$ is analytic. The map $E$ has a Siegel disc 
$S=\Phi(\mathbb{D})$ centered at 0 with a critical point $-1 \in \partial S$. By the same arguments as in the previous sections, the map $E$ has growth order 1 and an exceptional value 0 which is only assumed in 0 . Taking everything together we see that $E(z)=\lambda z e^{z}$. As the image of $\mathbb{D}$ under the quasiconformal map $\Phi$, the Siegel disc $S$ is a quasidisc as claimed.

Theorem 5.5. If $\rho \in \mathbb{R} \backslash \mathbb{Q}$ is of constant type, $\lambda=e^{2 \pi i \rho}$ and $d \geq 1$, then the Siegel disc of $P_{\lambda, d}(z)=\lambda z(1+z / d)^{d}$ is a quasidisc with the critical point $-d /(d+1)$ on the boundary.

Proof. The proof is exactly the same as above, the only difference being the particular mappings. Start with

$$
B_{a}(z)=e^{2 \pi i a} z^{d+1}\left[\frac{z+(2 d+1)}{1+(2 d+1) z}\right]^{d}
$$

where $a \in \mathbb{R}$ is chosen such that $\rho\left(B_{a}\right)=\rho$. Again $B_{a}$ is an analytic circle homeomorphism with critical point $-1 \in \mathbb{T}$, so it is quasisymmetrically linearizable. As in the previous proof we paste a rotation inside $\mathbb{T}$ quasiconformally, get a quasiregular map $\tilde{B}$ with $\tilde{B}(0)=0$ satisfying the assumptions of the qc-lemma and finally an analytic map $P=\Phi \tilde{B} \Phi^{-1}$. We normalize $\Phi$ by $\Phi(0)=0, \Phi(\infty)=\infty$ and $\Phi(-(2 d+1))=-d$. The original map has $d+1$ poles at $\infty$ and $d$ poles at $-1 /(2 d+1)$. After pasting the rotation, the new map has only $d+1$ poles at $\infty$, so $P$ is a polynomial of degree $d+1$. By construction $P(0)=0, P^{\prime}(0)=\lambda$ and $P$ has a Siegel disc which is a quasidisc with a critical point $c=\Phi(-1)$ on the boundary. Also $P$ has $d$ zeros at $-d=\Phi(-(2 d+1))$. This determines $P$ uniquely as $P(z)=\lambda z(1+z / d)^{d}=P_{\lambda, d}(z)$.

Remark. With a bit more work one can show the corresponding result for the Arnold family: If the rotation number is of constant type, then the Arnold-Herman ring is a quasi-annulus containing a critical point in each boundary component. This involves some more analysis of the parameter space of the Arnold family, so we will not include a proof here.

\section{ACKNOWLEDGEMENT}

I would like to thank Walter Bergweiler, Nuria Fagella and Steffen Rohde for their encouragement and many helpful discussions.

\section{REFERENCES}

[Ah1] Ahlfors, L.V., Lectures on quasiconformal mappings, Van Nostrand, 1966. MR 34:336

[Ah2] Ahlfors, L.V., Conformal Invariants, Topics in Geometric Function Theory, McGraw-Hill, 1973. MR 50:10211

[Ar] Arnol'd, V.I., Small denominators. I: Mappings of the circumference onto itself., AMS Translations, Ser. 2, 46 (1965), 213-284.

[Ba] Baker I.N., Wandering domains for maps of the punctured plane, Ann. Acad. Sci. Fenn. Ser. A I, 12 (1987), 191-198. MR 89g:30046

[Be] Beardon, A.F., Iteration of Rational Functions, Springer-Verlag, 1991. MR 92j:30026

[BI] Bojarski, B. and Iwaniec, T., Analytical foundations of the theory of quasiconformal mappings in $\mathbb{R}^{n}$, Ann. Acad. Sci. Fenn. Ser. A I, 8 (1983), 257-324. MR 85h:30023

[CG] Carleson, L. and Gamelin, T.W., Complex Dynamics, Springer-Verlag, 1993. MR 94h:30033

[Do] Douady, A., Disques de Siegel et anneaux de Herman, Sém. Bourbaki, 39 (1986-87), 151172. MR 89g:30049 
[EL] Eremenko, A. E. and Lyubich, M. Yu., Dynamical properties of some classes of entire functions, Ann. Inst. Fourier, 42, 4 (1992), 989-1020. MR 93k:30034

[Fa1] Fagella, N., The Complex Standard Family, Preprint.

[Fa2] Fagella, N., Limiting Dynamics for the Complex Standard Family, Internat. J. Bifur. Chaos Appl. Sci. Engrg., 5 (1995), no. 3, 673-699. MR 96f:58129

[Ge1] Geyer, L., Quasikonforme Deformation in der Iterationstheorie, Diplomarbeit, TU Berlin, 1994.

[Ge2] Geyer, L., Linearization of structurally stable polynomials, Progress in holomorphic dynamics, Pitman Research Notes, 387, Longman, 1998, 27-30. MR 99m:58154

[GF] Grauert, H. and Fritzsche, K., Einführung in die Funktionentheorie mehrerer Veränderlicher, Springer-Verlag, 1974. MR 51:8448

[He1] Herman, M., Sur les conjugaison différentiable des difféomorphismes du cercle à des rotations, Publ. Math. IHES, 49 (1979), 5-234. MR 81h:58039

[He2] Herman, M., Conjugaison quasi-symmétrique des homéomorphismes analytiques du cercle a des rotations, Manuscript.

[Hi] Hinkkanen, A., Uniformly quasiregular semigroups intwo dimensions, Ann. Acad. Sci. Fenn. Ser. A I, 21 (1996), 205-222. MR 96m:30029

[Ke] Keen, L., Topology and growth of a special class of holomorphic self-maps of $\mathbb{C}^{*}$, Erg. Th. Dyn. Sys., 9 (1989), 321-328. MR 91b:30070

[Ko] Kotus, J., Iterated holomorphic maps on the punctured plane, Dynamical systems (Sopron, 1985), Lecture Notes in Econom. and Math. Systems, 287, Springer-Verlag, (1987), 10-28. MR 92i: 58152

[Kr] Kriete, H., Herman's proof of the existence of critical points on the boundary of singular domains, Progress in holomorphic dynamics, Pitman Research Notes, 387, Longman, 1998, 31-40. MR 99i:30042

[LV] Lehto, O. and Virtanen, K., Quasikonforme Abbildungen, Springer-Verlag, 1965. MR 32:5872

[Ma] Makienko, P., Iterations of analytic functions in $\mathbb{C}^{*}$ (Russian) Dokl. Akad. Nauk SSSR 297 (1987), no. 1, 35-37; translation in Soviet Math. Dokl. 36 (1988), no. 3, 418-420. MR 88m:30066

[Mi] Milnor, J., Dynamics in One Complex Variable: Introductory Lectures, SUNY Stony Brook Preprint 1990/5.

[MS] de Melo, W. and van Strien, S., One-Dimensional Dynamics, Springer-Verlag, 1993. MR 95a:58035

[PM1] Pérez-Marco, R., Solution complète au problème de Siegel de linéarisation d'une application holomorphe au voisinage d'un point fixe (d'aprés J.-C. Yoccoz), Sém. Bourbaki 44ème année, 753, (1991-92). MR 94g:58190

[PM2] Pérez-Marco, R., Sur les dynamiques holomorphes non linéarisables et une conjecture de V. I. Arnold, Ann. Sci. École Norm. Sup., 26 (1993), 565-644. MR 94h:30035

[Po] Pommerenke, Ch., Boundary Behavior of Conformal Maps, Springer-Verlag, 1992. MR 95b:30008

[Sh] Shishikura, M., On the quasiconformal surgery of rational functions, Ann. Sci. École Norm. Sup., 20 (1987), 1-29. MR 88i:58099

[St] Steinmetz, N., Rational iteration, de Gruyter Studies in Math., 16, 1993. MR 94h:30035

[Su] Sullivan, D., Conformal Dynamical Systems, Geometric Dynamics, Proc. Int. Symp., Rio de Janeiro/Brasil 1981, Lect. Notes Math. 1007, 725-752 (1983). MR 85m:58112

[Sw] Świątek, G., Remarks on critical circle homeomorphisms, Bol. Soc. Bras. Mat., 29 (1998), 329-351.

[Yo1] Yoccoz, J.-C., Théorème de Siegel, nombres de Brjuno et polynomes quadratiques, Asterisque, 231 (1995), 3-88. MR 96m:58214

[Yo2] Yoccoz, J.-C., Conjugaison des difféomorphismes analytiques du cercle, Preprint.

Universität Dortmund, FB Mathematik, LS IX, 44221 Dortmund, Germany

E-mail address: geyer@math.uni-dortmund.de 